This is a pre-print version.

The final version is available at:

https://doi.org/10.1016/j.compfluid.2020.104474 


\section{Graphical Abstract}

Field inversion for data-augmented RANS modelling in turbomachinery flows

Andrea Ferrero, Angelo Iollo, Francesco Larocca

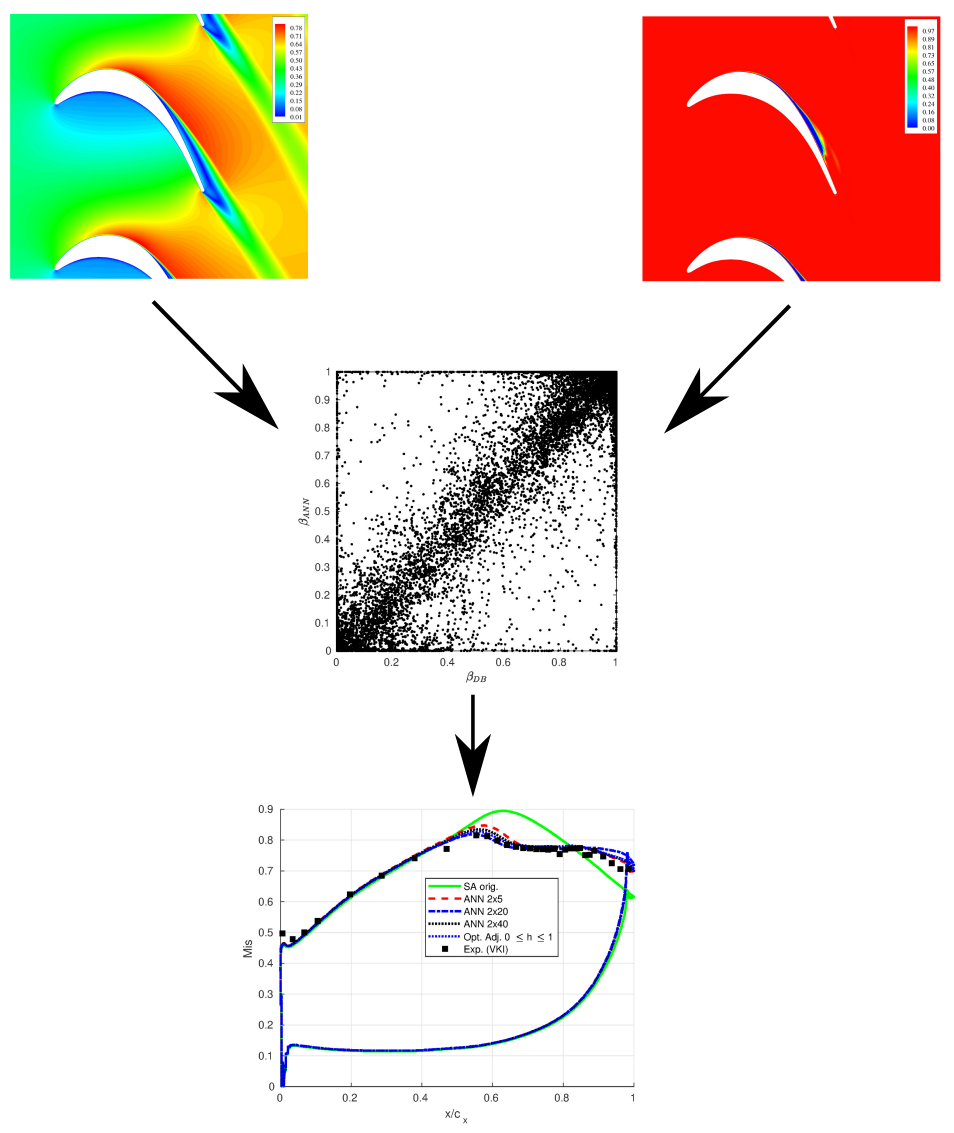




\section{Highlights}

- The field inversion approach is investigated for improving RANS models in turbomachinery flows

- Working conditions characterised by transition and separation are considered

- Some approaches to improve the robustness of the method are proposed

- The predictive ability of the method is investigated for several working conditions on different geometries 


\title{
Field inversion for data-augmented RANS modelling in turbomachinery flows
}

\author{
Andrea Ferrero ${ }^{\mathrm{a}, *}$, Angelo Iollo ${ }^{\mathrm{b}, \mathrm{c}, \mathrm{d}}$, Francesco Larocca ${ }^{\mathrm{a}}$ \\ ${ }^{a}$ Politecnico di Torino, 10129 Torino, Italy \\ ${ }^{b}$ Memphis Team, INRIA, F-33400 Talence, France \\ ${ }^{c}$ Univ. Bordeaux, IMB, UMR 5251, F-33400 Talence, France \\ ${ }^{d}$ CNRS, IMB, UMR 5251, F-33400 Talence, France
}

\section{Abstract}

Turbulence modelling in turbomachinery flows remains a challenge, especially when transition and separation phenomena occur. Recently, several research efforts have been devoted to the improvement of closure models for Reynolds Averaged Navier-Stokes (RANS) equations by means of machine learning approaches which make it possible to extract the knowledge hidden inside the available high-fidelity data (from experiments or from scale-resolving simulations). In this work the use of the field inversion approach is investigated for the augmentation of the Spalart-Allmaras RANS model applied to the flow in low pressure gas turbine cascades. As a first step, the field inversion method is applied to the T106c cascade at two different values of Reynolds number (80000-250000): an adjoint-based gradient method is employed in order to minimise the prediction error on the wall isentropic Mach number distribution. The data obtained by the correction field are then analysed by means of an Artificial Neural Network (ANN) which makes it possible to

\footnotetext{
${ }^{*}$ Corresponding author

Email address: andrea_ferrero@polito.it (Andrea Ferrero)
} 
generalise the correction by finding correlations which depend on physical variables. A study on the definition of the input variables and on the architecture of the ANN is performed. Different kind of corrections are evaluated and a particularly robust correction factor is obtained by limiting the range of the correction in the spirit of intermittency models. Finally, the ANN is introduced in an augmented version of the Spalart-Allmaras model which is tested on the T106c cascade (for values of the Reynolds number not considered during the training) and for the T2 cascade. The prediction ability of the method is investigated by comparing the numerical predictions with the available experimental data not only in terms of wall isentropic Mach number distribution (which was used as goal function during the field inversion) but also in terms of mass averaged exit angle and kinetic losses.

Keywords: Field inversion, Machine learning, Turbulence modelling, Turbomachinery

\section{1. Introduction}

2 The recent trends in the simulation of the flow field inside aerospace 3 propulsion systems are characterised by a growing interest towards high4 fidelity simulations which have become feasible thanks to a significant in5 crease in the available computational power. This paves the way to the 6 possibility of understanding complex physical effects which characterise tur7 bulence and combustion phenomena in modern engines. The ability to understand and control these effects can be exploited to increasing the performance 9 and reduce the emissions of existing propulsion systems.

However, scale-resolving simulations (like for example Direct Numerical Sim- 
ulations (DNS) or Large Eddy Simulations (LES)) cannot be easily integrated in the design process of industrial components. This is due to two main reasons: computational cost and difficulty to manage the results. It is clear that in the first steps of a design process several configurations must be investigated and the use of high-fidelity simulations in this phase would have a prohibitive cost. For this reason, less expensive approaches like RANS equations will be probably used for several years. As far as the management of the results is concerned, LES and DNS usually generate a huge amount of data for each simulation: in order to extract the useful information required by the design process it would be necessary to perform a complex post-process step. For example, even the computation of the average field from unsteady DNS data is not trivial because it is not known a-priori the extension of the time window required to get statistically converged results: several examples of low frequency phenomena which make difficult to compute the average field can be found in the literature, even looking to simple test cases, and special strategies to estimate the statistical error should be used (1). A review of the current state of the art for high-fidelity simulations in turbomachinery was proposed by Sandberg and Michelassi (2).

Recently, several research efforts have been devoted to the development of machine learning algorithms for all those applications in which a large amount of data must be processed. In particular, several recent works in the literature have been devoted to the use of machine learning techniques to analyse high-fidelity data from experiments or high-fidelity numerical simulations. The idea behind most of these recent works is to get the physical 
insight hidden in the data and use it to develop or improve low order datadriven models. An example of this philosophy is represented by the work of Xie et al. (3) who proposed a filtered reduced order model with a datadriven closure. Dupuis et al.(4) proposed an approach in which traditional surrogate models and machine learning are combined to improve the prediction of the flow on airfoils which work in subsonic or transonic conditions. Margheri et al.(5) performed a study on the epistemic uncertainty of some popular RANS models and used a generalised Polynomial Chaos response surface to perform the calibration of the model coefficients in the spirit of data assimilation strategies. In (6) the Proper Orthogonal Decomposition approach is used in a discontinuous Galerkin (DG) finite element framework (7) together with a domain decomposition strategy (8) to learn empirical local bases which are used to reduce the simulation cost of the flow field in gas turbines.

An alternative path was followed by Raissi and Karniadakis (9) who proposed an approach to identify the partial differential equations which govern a set of data: they applied the algorithm to an example in which they recovered the Navier-Stokes equations used to generate the database but the same approach could be used on experimental data to recover turbulence models. While the work of Raiss and Karniadakis (9) aims at discovering the full governing model, several works focus on the improvement of existing models. For example, Wang et al. (10) developed a machine learning strategy to predict the discrepancy in RANS modelled Reynolds stresses starting from DNS data. Weatheritt et al.(11) proposed the use of Gene Expression Programming to identify new expressions for the stress-strain relationship. Promising 
results were obtained with this technique on high pressure turbines (12).

Duraisamy et al. $(13 ; 14)$ proposed a strategy based on field inversion and machine learning which allows to improve the prediction ability of RANS models. This approach is exploited in the present work in order to improve RANS modelling for low pressure gas turbine cascades.

Machine learning techniques have been investigated also on multiphase flows $(15 ; 16)$, combustion $(17 ; 18 ; 19)$ and engine modelling $(20 ; 21)$. Finally, a comprehensive review of the machine learning techniques proposed for the improvement of turbulence modelling can be found in (22).

The paper is organised as follows. In Section 2 the original RANS model is presented. In Section 3 the methods used for the discretisation of the equations are described. In Section 4 the field inversion approach is described and it is then applied to the T106c gas turbine cascade in Section 5. The data obtained by the field inversion are analysed by means of machine learning techniques in Section 6 in order to generalise the obtained results. Finally, the improved RANS model is tested on the T106c and on the T2 cascades in Section 7.

\section{Physical model}

This work is devoted to the prediction of the compressible turbulent flow in 2D turbine cascades. The study starts from the Spalart-Allmaras (SA) model implemented for compressible equations, following the guidelines of (23). This model is widely used in the literature for fully turbulent flows. However, the model is not suitable for the prediction of transitional flows at low Reynolds numbers. The original model gives the possibility to impose 
the transition location (by means of the trip term $f_{t 1}$ defined in $(23)$ ) but this choice is rarely followed in the literature because in general the location of transition in not known a-priori. Furthermore, when the transition trip term $f_{t 1}$ is used a second term $f_{t 2}$ for delaying natural transition (and making the trip term $f_{t 1}$ effective) is also activated. Further details on the effects of the term $f_{t 2}$ in the prediction of the flow around the T106c cascade can be found in $(24)$.

In the present work the SA model is used without the trip terms $f_{t 1}$ and $f_{t 2}$. With this choice the model is expected to work fine for high Reynolds numbers but to fail in predicting transition and separation at low values of Reynolds number. This model tends indeed to produce an excessive amount of turbulent eddy viscosity on this kind of flows (24). For this reason, it represents an optimal baseline for testing the field inversion approach and evaluating how much the original model can be improved.

The mass-averaged RANS equations are reported in the following:

$$
\begin{gathered}
\frac{\partial \rho}{\partial t}+\nabla \cdot(\rho \boldsymbol{u})=0 \\
\frac{\partial}{\partial t}(\rho \boldsymbol{u})+\nabla \cdot(\rho \boldsymbol{u} \boldsymbol{u})=-\nabla p+\nabla \cdot \boldsymbol{\tau} \\
\frac{\partial E}{\partial t}+\nabla \cdot(\boldsymbol{u}(E+p))=\nabla \cdot(\tau \cdot \boldsymbol{u}-\boldsymbol{q}) \\
\frac{\partial \rho \hat{\nu}}{\partial t}+\nabla \cdot(\rho \boldsymbol{u} \hat{\nu})=\rho(P-D)+\frac{1}{\sigma} \nabla \cdot(\rho(\nu+\hat{\nu}) \nabla \hat{\nu})+\frac{c_{b 2}}{\sigma} \rho(\nabla \hat{\nu})^{2}-\frac{1}{\sigma}(\nu+\hat{\nu}) \nabla \rho \cdot \nabla \hat{\nu}
\end{gathered}
$$


where $\rho, \boldsymbol{u}, p, E, \nu, \hat{\nu}, \boldsymbol{x}$ and $t$ are density, velocity, pressure, total energy per unit volume, molecular viscosity, modified eddy viscosity, spatial position and time, respectively. A fluid with constant specific heat ratio $\gamma$ and constant viscosity is considered. The following equation for the energy is considered:

$$
E=\frac{P}{\gamma-1}+\frac{1}{2} \rho \boldsymbol{u} \cdot \boldsymbol{u}
$$

where $\gamma$ is the specific heat ratio.

The viscous stress tensor $\boldsymbol{\tau}$ includes both the molecular and eddy viscosity contributions and its components are given by:

$$
\tau_{i j}=2 \rho\left(\nu+\hat{\nu} f_{v 1}\right)\left(\frac{1}{2}\left(\frac{\partial u_{i}}{\partial x_{j}}+\frac{\partial u_{j}}{\partial x_{i}}\right)-\frac{1}{3} \frac{\partial u_{k}}{\partial x_{k}} \delta_{i j}\right)
$$

The production $P$ and destruction $D$ terms in Eq. 4 are computed as follows:

$$
P=c_{b 1} \tilde{S} \tilde{\nu} \quad D=c_{w 1} f_{w}\left(\frac{\tilde{\nu}}{d}\right)^{2}
$$

with the following definitions:

$$
\begin{gathered}
f_{w}=g\left(\frac{1+c_{w 3}^{6}}{g^{6}+c_{w 3}^{6}}\right)^{1 / 6} g=r+c_{w 2}\left(r^{6}-r\right) \quad r=\min \left(\frac{\tilde{\nu}}{\tilde{S}^{2} \kappa^{2} d^{2}}, r_{l i m}\right) \\
\tilde{S}= \begin{cases}S+\bar{S} & \text { if } \bar{S}>=-c_{v 2} S \\
S+\frac{S\left(c_{v 2}^{2} S+c_{v 3} \bar{S}\right)}{\left(c_{v 3}-2 c_{v 2}\right) S-\bar{S}} & \text { if } \bar{S}<-c_{v 2} S\end{cases}
\end{gathered}
$$

110 where $S$ is the vorticity magnitude and $\bar{S}$ is:

$$
\bar{S}=\frac{\tilde{\nu}}{\kappa^{2} d^{2}} f_{v 2}
$$

111 The functions $f_{v 1}$ and $f_{v 2}$ depend on the viscosity ratio $\chi=\frac{\tilde{\nu}}{\nu}$ :

$$
f_{v 1}=\frac{\chi^{3}}{\chi^{3}+c_{v 1}^{3}} \quad f_{v 2}=1-\frac{\chi}{1+\chi f_{v 1}}
$$


The constants $\sigma, c_{b 1}, c_{b 2}, c_{v 1}, c_{w 1}$ are defined in (23).

Finally, the heat flux $\boldsymbol{q}$ is described by the Fourier's law:

$$
\boldsymbol{q}=-\left(\frac{c_{p} \mu}{\operatorname{Pr}}+\frac{c_{p} \rho \hat{\nu} f_{v 1}}{P r_{t}}\right) \nabla T
$$

where $T, c_{p}, \operatorname{Pr}$ and $\operatorname{Pr}_{t}$ are the temperature, the constant pressure specific heat capacity, the Prandtl number and the turbulent Prandtl number. The test cases considered in this work refer to experiments performed with air and so the following values are assumed: $\gamma=1.4, \operatorname{Pr}=0.72$ and $P r_{t}=0.9$.

\section{Implicit Discontinuous Galerkin discretization}

The discontinuous Galerkin (DG) scheme is used in this work for the spatial discretisation on the governing equations. This approach is characterised by a significant flexibility since it allows to easily manage high-order reconstructions on unstructured meshes. The main idea behind this kind of scheme consists in adopting an high-order polynomial reconstruction inside each element without any continuity constraint at the interface between different elements. As a result, the scheme can be easily exploited in the framework of automatic adaptive approaches, in which both the size (h-adaptivity, $(25 ; 26 ; 27 ; 28))$, the order (p-adaptivity $(29 ; 30 ; 31))$ or both properties (hp-adaptivity, $(32 ; 33 ; 34))$ can be locally adapted following some error indicators.

The computational domain $\Omega$ is discretised with a hybrid mesh which contains a structured boundary layer mesh close to the body surrounded 
by an unstructured mesh. The grid is generated by means of Gmsh (35) with the Frontal-Delaunay for Quads algorithm. The management of the unstructured grid in the parallel MPI environment is performed through the DMPlex class (36) provided by the PETSc library (37).

The numerical approximation of the $l$-th conservative variable $u_{l}(\boldsymbol{x}, t)$ inside each element $\Omega_{e}$ is described by a modal basis with size $N_{e}=\frac{(k+1)(k+2)}{2}$ with a reconstruction order $k$ :

$$
u_{l}(\boldsymbol{x}, t)=\sum_{i=1}^{N_{e}} \tilde{u}_{l i}(t) \phi_{i}(\boldsymbol{x}) \quad 1 \leq i \leq N_{e}
$$

where $\tilde{u}_{l i}(t) \in \mathbb{R}^{N_{e}}$ contains the degrees of freedom inside the element for the $l$-th conservative variable. The basis functions $\phi_{i}(\boldsymbol{x})$ are obtained by the modified Gram-Schmidt orthonormalisation applied to a set of monomials defined in the physical space, following the approach of Bassi et al. (38). In this work a third order accurate DG scheme is used $\left(k=2, N_{e}=6\right)$.

The spatial discretisation is completed by a projection of the governing equation on the space of the approximation functions. The resulting weak formulation consists in a set of ordinary differential equations in time. The convective terms which appear in the numerical fluxes at the interface between the elements are evaluated by means of an approximate Riemann problem solver (following (39) and (40)). Diffusive terms are evaluated by means of a recovery-based approach (41).

Time integration is here performed by means of the linearised implicit Euler method. Since steady problems are considered the use of a first order time integrator appears suitable since it does not influence the accuracy of the final steady solution and it has good dissipative properties which are useful to accelerate the numerical transients. The solution of the linear system which 
is obtained at each time step is performed in parallel by means of the GMRES algorithm with the additive Schwarz preconditioner provided by the PETSc library (37). The GMRES algorithm is employed by setting the maximum number of iterations to 200, the dimension of the Krylov subspace to 100 and the absolute tolerance to $10^{-12}$. The CFL number which controls the time step size is automatically adjusted according to the evolution of the residuals following the pseudo-transient continuation strategy (42). In particular, the CFL number is allowed to vary between $10^{2}$ and $10^{4}$. During the first steps of the transient, a feedback filtering procedure (43) is applied to remove potential instabilities which can appear due to the large CFL number. This filtering procedure is deactivated when the residuals drop under a certain threshold and so it does not influence the steady solution.

\section{Field inversion and machine learning in a DG framework}

The field inversion approach proposed by (14) requires to define a goal function $G$ which measures the distance between the experimental data and the predicted numerical results. The procedure requires the solution of an optimisation problem in which a field $\beta(x)$ is found in order to minimise the goal function $G$. The field $\beta(x)$ is then introduced in a correction term $h(\beta(x))$ which multiplies the production term in the SA transport equation:

$$
\frac{\partial \rho \hat{\nu}}{\partial t}+\nabla \cdot(\rho \boldsymbol{u} \hat{\nu})=\rho[h(\beta) P-D]+\frac{1}{\sigma} \nabla \cdot(\rho(\nu+\hat{\nu}) \nabla \hat{\nu})+\frac{c_{b 2}}{\sigma} \rho(\nabla \hat{\nu})^{2}-\frac{1}{\sigma}(\nu+\hat{\nu}) \nabla \rho \cdot \nabla \hat{\nu}
$$

In the original works of $(14 ; 44)$ the correction was chosen as $h(\beta)=\beta$. In this work, different choices are investigated for the function $h(\beta)$, as described 
in the next section.

As far as the goal function $G$ is concerned, the following choice is made:

$$
G=\int_{w}\left(M_{s}-M_{s}^{e x p}\right)^{2} d l+\lambda \int_{\Omega}(\beta-1)^{2} d \Omega
$$

The first term is a line integral performed on the wall of the blade and allows to evaluate the norm-2 error on the wall isentropic Mach number distribution $M_{s}$, which is defined as:

$$
M_{s}=\sqrt{\frac{2}{\gamma-1}\left[\left(p_{i}^{0} / p_{w}\right)^{(\gamma-1) / \gamma}-1\right]}
$$

where $p_{w}$ is the static pressure at wall and $p_{i}^{0}$ is the inlet total pressure.

The second term is a surface integral on the computational domain $\Omega$ which acts as a Tikhonov regularisation (45): it penalises the goal function when the correction factor is far from 1. This is useful to avoid unnecessary corrections which could be introduced during the optimisation process but which are not required in the final optimal solution. The choice of the penalisation constant $\lambda$ will be discussed in the next Section.

In order to solve the optimisation problem, a simple gradient descent method is applied. The field $\beta$ will be described in terms of the same basis functions used for the conservative variables. Starting from the original SA model $(h(\beta(x))=1)$ the degrees of freedom related to the field $\beta$ are updated with the gradient descent method:

$$
\tilde{\beta}=\tilde{\beta}-\delta \frac{d G}{d \tilde{\beta}}
$$

where $\delta$ is the step size that in this work is chosen constant for simplicity $(\delta=0.1)$. 
Since the dimension of the optimisation problem is related to the total number of degrees of freedom per equation the computation of the gradient $\frac{d G}{d \tilde{\beta}}$ by means of numerical differentiation would be prohibitive. For this reason, an adjoint-based gradient evaluation was implemented. The gradient of the goal function $G$ which respect to the degrees of freedom of the field $\beta(x)$ is computed as:

$$
\frac{d G}{d \tilde{\beta}}=\frac{\partial G}{\partial \tilde{\beta}}+\psi^{T} \frac{\partial R}{\partial \tilde{\beta}}
$$

where $R$ represents the residual of the governing equations. The first term contains only the contributions related to the penalisation integral which appears in the goal function. The adjoint variable $\Psi$ is computed by the solution of the following linear system with the GMRES iterative solver:

$$
\left[\frac{\partial R}{\partial \tilde{u}}\right]^{T} \Psi=-\left[\frac{\partial G}{\partial \tilde{u}}\right]^{T}
$$

in which the jacobian matrix $\left[\frac{\partial R}{\partial \tilde{u}}\right]$ is already available from the implicit time integrator and the term $\left[\frac{\partial G}{\partial \tilde{u}}\right]$ contains the derivatives of the goal function with respect to the fluid dynamics degrees of freedom. This last term was computed by means of automatic differentiation with the Tapenade tool (46). Summarising, the procedure works as follows. First of all, a steady solution with the original SA model is obtained. The solution is considered steady when the residuals of all the governing equations are lower than $10^{-6}$. Usually, the SA equation is the one which converges with the lowest speed so when the condition is satisfied the residuals of the Eqs. 1-3 are orders of magnitudes lower (typically around $10^{-8}-10^{-10}$ ). When the steady solution is reached, the gradient $\frac{d G}{d \tilde{\beta}}$ is computed by the adjoint approach and the correction field is updated. This generates a transient which is solved in 
time up to a new steady solution. Since the perturbation introduced by the correction update is small, the transient can be easily solved by marching in time with a very large CFL number. For example, in this work the constant value $\mathrm{CFL}=5000$ is used for this part of the computation. The procedure is repeated until the goal function does not show any significant improvement.

The correction field $h(\beta(x))$ obtained by the inversion process can be exploited for different purposes. On one hand, it gives insight for the development of new turbulence models since it shows where and how the original model fails. On the other hand, it is possible to directly generalise the correction in order to obtain a new model which can be used for predictive simulations. For example, Duraisamy and Durbin (47) used the results of field inversion to define a transport equation for an intermittency factor, where the different terms of the transport equation are computed by means of machine learning techniques. Alternatively, it is possible to find a local closure which allows to define the correction field as a function of local physical quantities $(14 ; 44)$. This last approach is followed in the present work. In particular, the results of the inverse problem will be exploited to train an Artificial Neural Network (ANN) which can then be used to define an augmented version of the SA model.

\section{Field inversion on the T106c cascade}

The field inversion approach is here applied to the flow around the T106c gas turbine cascade. This profile is representative of high-lift low pressure gas turbines in modern turbofan engines. The cascade was experimentally 
investigated at the VKI and some experimental results are available from the literature $(48 ; 49 ; 50)$. In particular, the wall isentropic Mach number distribution, the mass averaged kinetic losses and exit angle in the wake are available for several values of the Reynolds number. The flow field is studied for an inlet angle $\alpha=32.7^{\circ}$, an isentropic exit Mach number $M_{2 s}=0.65$ and different values of the exit isentropic Reynolds number $8 \cdot 10^{4} \leq R e_{2 s} \leq$ $2.5 \cdot 10^{5}$. The Reynolds number $R e_{2 s}$ is defined by using the blade chord and the isentropic exit velocity and density. The dynamic viscosity is assumed constant. The turbulence intensity during the experiments was very low (0.9\%): for this reason all the RANS simulations are performed by setting a very small value of inlet eddy viscosity $(\tilde{\nu} / \nu=0.1)$.

Houmorziadis (51) showed that the Reynolds number in low pressure gas turgines of turbofan engines is the range between $10^{5}-4 \cdot 10^{5}$ where the smaller values are observed in cruise conditions and the higher values are obtained at take-off. The high-lift profiles can show large laminar separations at low values of Reynolds number. When the Reynolds number is increased the separation transforms from an open separation to a closed separation in which there is a separation bubble followed by reattached flow. The evolution from one configuration to the other takes place in a small range of Reynolds number and so the flow is quite sensitive to the working condition.

The presence of separation can be easily noticed in the experimental studies on these flows by checking the wall isentropic Mach number distribution: the separation is usually related to the presence of a plateau in the distribution. Singh et al.(44) showed that the wall pressure distribution (which is directly related to the isentropic Mach number distribution) can be effectively used 
in the field inversion approach for improving the prediction of separated flows. They indeed showed that the field inversion based on the wall pressure distribution can significantly improve the prediction of the Reynolds stresses in the separation region (44). For these reasons, the field inversion algorithm used in this work will use the error on the wall isentropic Mach number distribution as goal function.

First of all, a convergence study is performed on the T106c cascade with the original SA model at the highest Reynolds number $\left(R e_{2 s}=2.5 \cdot 10^{5}\right)$. Three different meshes and two reconstruction orders $(1 \leq k \leq 2)$ are evaluated. The convergence level is assessed by checking the mass averaged value of the kinetic losses in a control section located $0.465 c_{x}$ behind the trailing edge. The kinetic losses are defined in the following way:

$$
\zeta=1-\frac{1-\left(p_{e} / p_{e}^{0}\right)^{(\gamma-1) / \gamma}}{1-\left(p_{e} / p_{i}^{0}\right)^{(\gamma-1) / \gamma}}
$$

where $p_{e}, p_{e}^{0}$ and $p_{i}^{0}$ are the static pressure in the control section, the total pressure in the control section and the inlet total pressure, respectively. The results of the convergence analysis are reported in Table 1 which shows the number of elements $n_{\text {ele }}$, the number of degrees of freedom per equation $n_{D O F}$ and the predicted averaged losses. It is useful to remember that in the asymptotic range mesh refinement gives a fixed convergence order (depending on $k$ ) while order refinement gives exponential convergence.

We emphasise that the losses in the wake represent a better goal function for the convergence assessment with respect to the wall isentropic Mach number distribution because the original SA model over-predicts significantly the turbulence eddy viscosity and so it gives a wall isentropic Mach number distribution which is very similar to what would be obtained by an inviscid 
Euler simulation, regardless of the mesh resolution. In contrast, the wake losses are influenced by the mesh resolution in the boundary layer and in the wake region.

The mesh $\mathrm{C}$ reported in Tab.1 will be used for all the following simulations with a third order accurate DG scheme $(k=2)$. The mesh contains 40436 elements and so the total number of degrees of freedom per equation is equal to 242616. The dimensionless wall cell size is $y^{+}<1$ on the entire surface.

\begin{tabular}{|l|c|c|c|}
\hline & $n_{\text {ele }}$ & $n_{D O F}$ & $\zeta$ \\
\hline Mesh A, k=1 & 11480 & 34440 & $2.39 \mathrm{E}-002$ \\
\hline Mesh B, k=1 & 21195 & 63585 & $2.27 \mathrm{E}-002$ \\
\hline Mesh C, k=1 & 40436 & 121308 & $2.24 \mathrm{E}-002$ \\
\hline Mesh A, k=2 & 11480 & 68880 & $2.25 \mathrm{E}-002$ \\
\hline Mesh B, k=2 & 21195 & 127170 & $2.24 \mathrm{E}-002$ \\
\hline Mesh C, k=2 & 40436 & 242616 & $2.24 \mathrm{E}-002$ \\
\hline
\end{tabular}

Table 1: Mass averaged kinetic losses: convergence with grid size and reconstruction order

As reported in Equation 14, the field inversion approach requires to alter the production term by the presence of the correction factor $h(\beta)$. In this work, different expressions for $h(\beta)$ are investigated. The most straightforward approach, which was used by Singh et al. (44) for the study of wind turbine airfoils, consists in setting :

$$
h(\beta)=\beta \quad \beta \in \mathbb{R}
$$

In this way the correction factor is free to assume both positive and negative values and so the correction term is very general. However, this generality 
comes with a price: since $h(\beta)$ is not limited it can lead to unstable numerical results during the transients which must be solved in predictive simulations. An alternative approach, experimented in this work, consists in setting

$$
h(\beta)=\beta^{2} \quad \beta \in \mathbb{R}
$$

In this way the correction term is not allowed to assume negative values. This means that the generality of the approach is reduced but the robustness of the simulation is increased because the correction term cannot change the nature of the production term (it can, in the limit, set the production to zero but it cannot transform the production term into a destruction term).

A third approach, which showed the most robust results in this work, is reported in the following. The idea behind this approach is to mimic the behaviour of intermittency models in which the production term of the RANS model is reduced by a factor defined in the range $[0,1]$ in order to reproduce transition phenomena. Following this approach, the correction term is defined as a smooth ramp function of $\beta$ :

$$
h(\beta)= \begin{cases}0 & \text { if } \beta \leq 0 \\ 3 \beta^{2}-2 \beta^{3} & \text { if } 0<\beta<1 \\ 1 & \text { if } \beta \geq 1\end{cases}
$$

This last approach is the least general between the three alternatives examined in this work but it is the most robust. This is due to the fact that, in the end, the correction factor $h$ will be expressed by means of an ANN. When the SA model augmented by the ANN correction term will be used for 
actual predictions, the ANN will be asked to compute the correction factor for input values which could be outside of the range explored in the training database. This is very likely to happen during the numerical transient which must be solved before getting the steady solution. However, ANNs are known for their poor extrapolation accuracy and so the use of a more general expression (like for example the one defined by Equation 21) would allow the presence of unlimited values of the correction factor. In contrast, when the correction factor is limited in the range $0 \leq h \leq 1$ the model can behave, in the limit, as the original SA model (when $h \rightarrow 1$ ) or as the laminar Navier-Stokes equations (when $h \rightarrow 0$ ).

In order to understand whether the limitation introduced by Equation 23 affects the ability of the field inversion to match the experimental data, the different definitions of $h(\beta)$ are tested on the T106c cascade. In particular, the gradient based optimisation process is carried out for the T106c at $R e_{2 s}=8 \cdot 10^{4}$ and $R e_{2 s}=2.5 \cdot 10^{5}$. The plot in Figure 1 shows the history of the goal function during the optimisation process. The results shows that after approximately 50 steps of the gradient descent algorithm a minimum is reached. This optimisation is carried out by starting from the original SA model with $h=1$ in all the domain and using the unlimited correction factor defined by Eq. 21 with $\lambda=0$.

The optimal field obtained from this first step is then used as initial field for a second optimisation in which the correction factor is limited according to Eq. 23. It is useful to emphasise that, in order to apply the correction factor defined by Eq. 23, it is not possible to start with a uniform field with $\beta=1$. 
This is due to the fact that the derivative of the smooth ramp function is null for $\beta=1$ and so it would not be possible to update the solution since the gradient of the goal function would remain to zero according to Eq. 18 $\left(\frac{\partial R}{\partial \tilde{\beta}}=\frac{\partial R}{\partial g} \frac{\partial g}{\partial \tilde{\beta}}\right.$, with $\frac{\partial g}{\partial \tilde{\beta}}=0$ for $\left.\beta=1\right)$.

In order to compare the two approaches, the wall isentropic Mach number distribution is reported in Figure 2 for the original SA model and the optimised solutions related to Eq. 21 and 23. The results for the correction factor defined by Eq.22 are not reported in the plot since they overlap the other results related to 21 and 23. The Figure shows also the available experimental data which are used to drive the optimisation process. The optimal solutions show a good match with the experimental data and a significant improvement with respect to the baseline model. This test confirms that the limited correction factor defined by Eq. 23 is able to provide an optimal solution which is comparable to the results provided by the unlimited correction factor. This is due to the fact that the original SA model overestimates significantly the turbulence production in this kind of flows and so the use of a correction factor limited between 0 and 1 is sufficient to correct the model. In this sense, the correction factor proposed in this work acts exactly as a intermittency correction in the framework of laminar-to-turbulence transition. After this analysis, the limited correction factor defined by Eq. 23 was chosen for all the following simulations.

The plots in Figure 3 show the Mach field for the original SA model and optimal model at $R e_{2 s}=8 \cdot 10^{4}$ and $R e_{2 s}=2.5 \cdot 10^{5}$. The optimal solution at $R e_{2 s}=8 \cdot 10^{4}$ is characterised by a large open separation which is completely missed by the original SA model. The optimal solution at $R e_{2 s}=2.5 \cdot 10^{5}$ 
shows a small separation bubble followed by reattachment. Again, this separation is missed by the original SA model.

Finally, the correction field at $R e_{2 s}=8 \cdot 10^{4}$ and $R e_{2 s}=2.5 \cdot 10^{5}$ for the case defined by Eq. 23 is reported in Figures 4 and 5 for $\lambda=0$ and $\lambda=10^{-3}$, respectively. An analysis of the pictures shows clearly that the adjoint approach obtained an optimal solution in which the production term is deactivated in the boundary layer for the first portion of the suction side: the algorithm has recovered a laminar separation just by using the knowledge on the experimental wall isentropic Mach number distribution. As far as the influence of $\lambda$ is concerned, a study with $\lambda=0,10^{-2}, 10^{-3}, 10^{-4}$ is performed. These values are chosen by running a preliminary simulation with $\lambda=0$ and then evaluating the order of magnitude of the two integrals which appear in the goal function defined by Eq. 15. For all these values, the optimal wall isentropic Mach number distribution does not show significant variations. The weak influence of the parameter $\lambda$ can be seen in Figures 4 and 5 where the higher value of $\lambda$ tends to avoid unnecessary corrections at the end of the separation region.

\section{Machine learning on the T106c cascade}

The field inversion algorithm described in the previous section is able to provide a correction field which alters the original SA model in order to match very well the experimental results for two different working conditions. In this section this result will be generalised in order to express the correction factor as a function of some physical features. In particular, several choices 


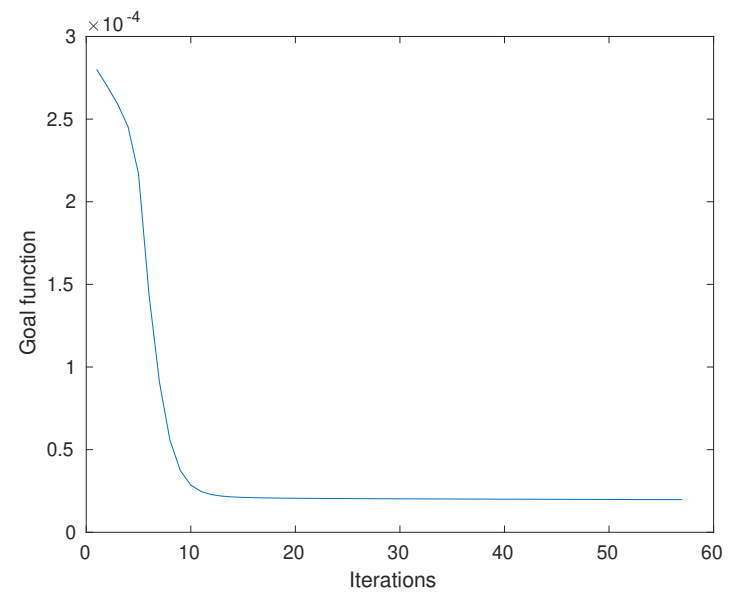

Figure 1: Adjoint-based optimization history for T106c at $R e_{2 s}=8 \cdot 10^{4}$ with $\beta \in R$

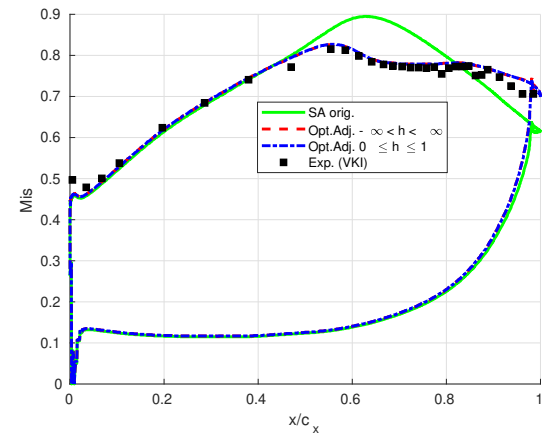

(a)

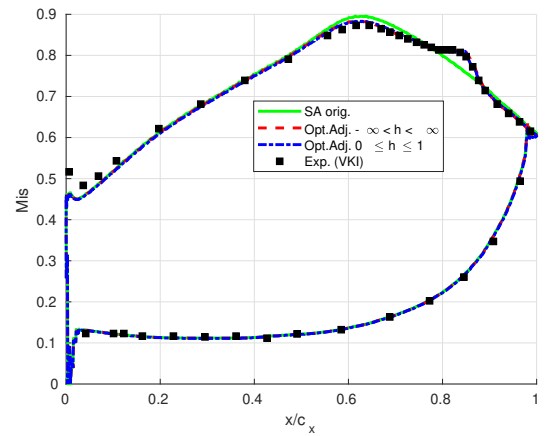

(b)

Figure 2: Comparison between original SA model, optimized model and experimental results in terms of Mis distribution for the T106c at $R e_{2 s}=8 \cdot 10^{4}$ (a) and $R e_{2 s}=2.5 \cdot 10^{5}$ (b) 


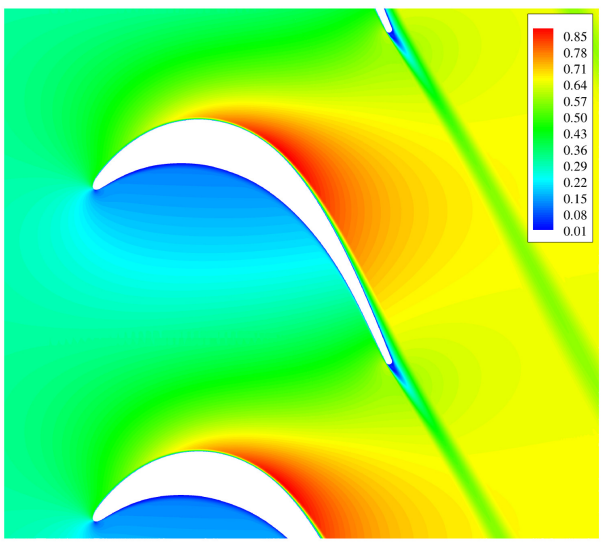

(a)

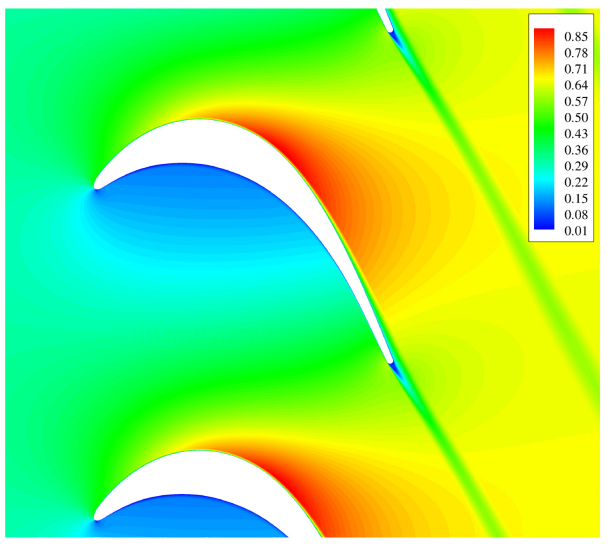

(c)

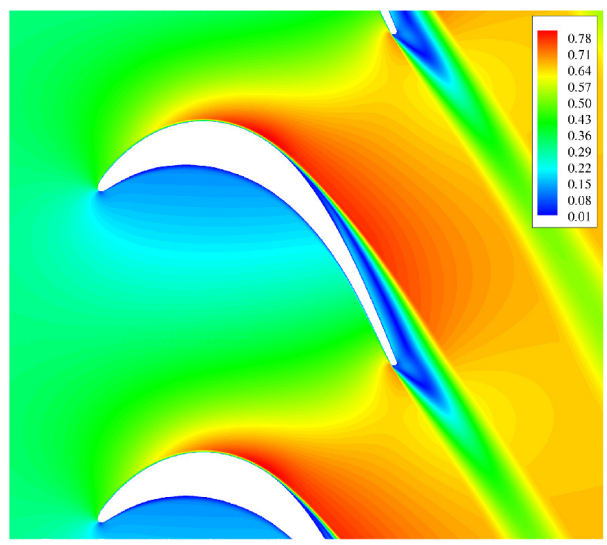

(b)

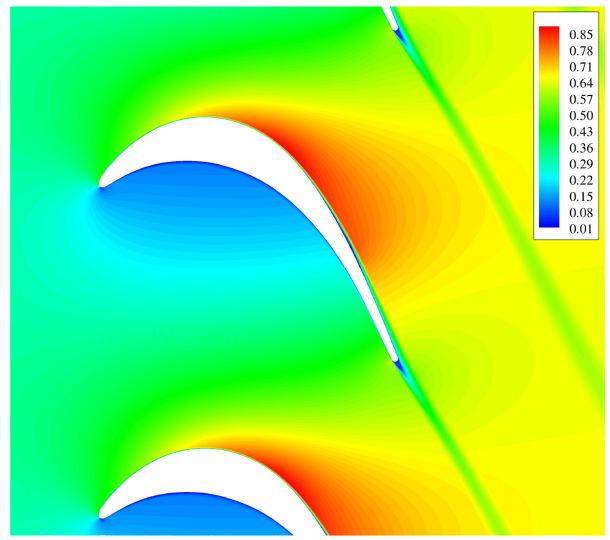

(d)

Figure 3: Mach field for T106c with the original SA model (a,c) and with optimised model $(\mathrm{b}, \mathrm{d})$ at $R e_{2 s}=8 \cdot 10^{4}(\mathrm{a}, \mathrm{b}) R e_{2 s}=2.5 \cdot 10^{5}(\mathrm{c}, \mathrm{d})$ 


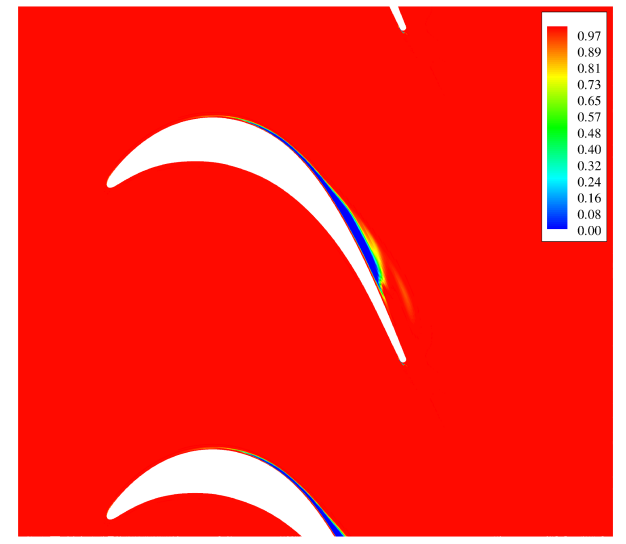

(a)

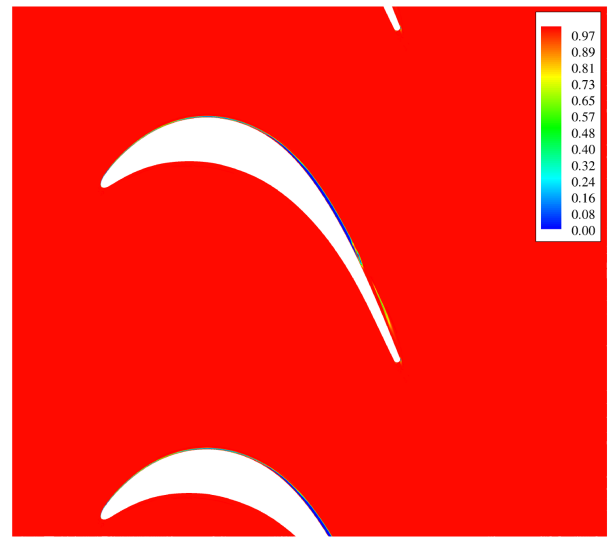

(b)

Figure 4: Correction field $h(x)$ for T106c at $R e_{2 s}=8 \cdot 10^{4}$ (a) and $R e_{2 s}=2.5 \cdot 10^{5}$ (b) with $\lambda=0$

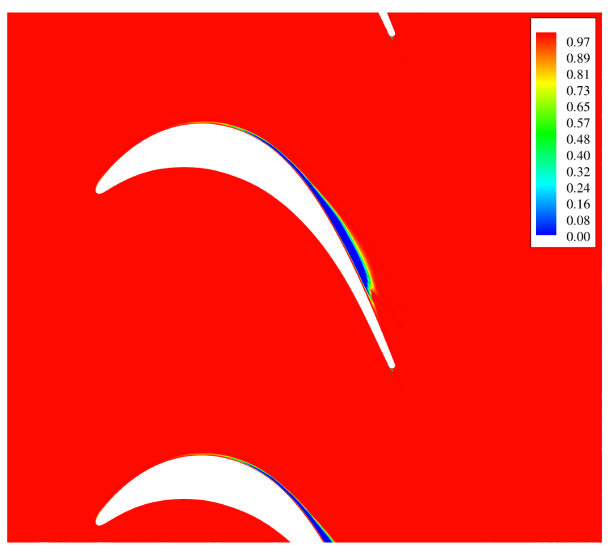

(a)

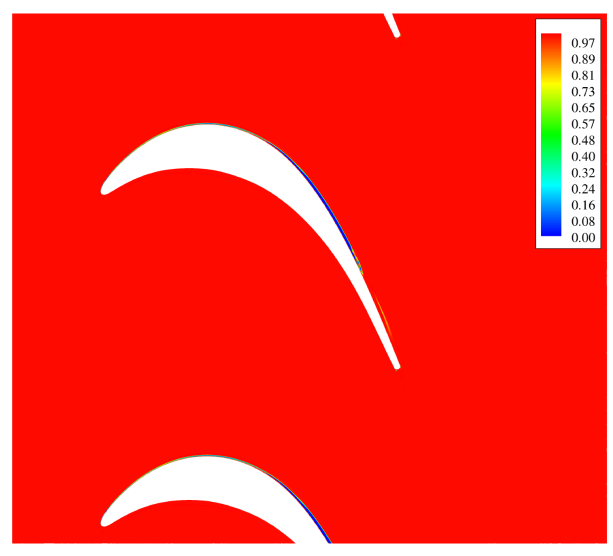

(b)

Figure 5: Correction field $h(x)$ for T106c at $R e_{2 s}=8 \cdot 10^{4}$ (a) and $R e_{2 s}=2.5 \cdot 10^{5}$ (b) with $\lambda=10^{-3}$ 
related to the inputs and the architecture of the ANN used to express the correction factor will be investigated.

\subsection{Choice of the inputs}

The choice of the input variables of the ANN is not a trivial task. In particular, it is necessary to avoid input variables which would introduce a dependency on the particular frame of reference which is used to study the problem (i.e. Galilean invariance must be satisfied). Furthermore, there should not be strong correlations between the different input variables and they should be chosen as adimensional quantities in order to get general results.

A natural choice is to identify some adimensional groups which appear in the source term of the original RANS model and use them as input for the ANN. This choice was for example carried out by Singh et al. (44).

A similar approach is used in this work but particular attention is here devoted to the robustness and the prediction ability of the model. The following five input variables are used: $\chi, \log \left(\tau / \tau_{\text {ref }}+\epsilon\right), f_{d}^{\prime}, \log (P /(D+\epsilon)+\epsilon)$ and $\log (|\nabla \tilde{\nu}| d /(\nu+\tilde{\nu})+\epsilon)$. The plots in Figure 6 show the distribution for all the inputs variables in the optimised solution at $R e_{2 s}=8 \cdot 10^{4}$.

The first input, $\chi$, simply represents the turbulent intensity. The quantity $\tau / \tau_{\text {ref }}$ is obtained by normalising the module of the stress tensor with respect to a reference stress. The reference stress is here defined as $\tau_{\text {ref }}=\rho(\nu+\tilde{\nu})^{2} / d^{2}$ which makes this input a local quantity. In contrast, Singh et al. (44) used a non local normalisation in which the stress tensor is normalised with respect to the wall stress $\tau_{w}$. However, such non-local terms are avoided 
in this work since the presence of non-local terms reduces significantly the scalability of the discretisation in a parallel environment. Furthermore, the physical meaning of using $\tau_{w}$ for the normalisation is clear for the mesh points in the boundary layer but is not so clear for other regions, like for example the wake. Finally, a logarithmic scaling of the quantity $\tau / \tau_{\text {ref }}$ was observed to significantly improve the fitting of the database. The additive constant $\epsilon=10^{-5}$ is introduced to prevent the algorithm of the logarithm to become null.

The term $f_{d}^{\prime}$ is introduced in this work as a modification of the term $f_{d}$ used by Singh et al. (44) and originally proposed by (52) in the framework of Detached Eddy Simulations. The terms are defined as:

$$
f_{d}=1-\tanh \left(\left(8 r_{d}\right)^{3}\right) \quad f_{d}^{\prime}=1-\tanh \left(\left(r_{d}\right)^{0.5}\right)
$$

where the quantity $r_{d}$ is an adimensional group obtained by combining wall distance, turbulence and molecular viscosity and velocity gradient:

$$
r_{d}=\frac{\nu+\tilde{\nu}}{d^{2} \kappa^{2} \sqrt{\frac{\partial u_{i}}{\partial x_{j}} \frac{\partial u_{i}}{\partial x_{j}}}}
$$

where $\kappa=0.41$ is the von Karman constant.

The plot in Figure 6 explains why in this work the term $f_{d}^{\prime}$ is used instead of $f_{d}$ : both terms are limited between 0 and 1 but $f_{d}^{\prime}$ allows to better describes the flow features close to wall while $f_{d}$ tends to compress the information and does not allow to distinguish the different structures. This qualitative analysis was confirmed by quantitative analysis which shows that an ANN with $f_{d}^{\prime}$ was able to better fit the database with respect to an equivalent ANN with $f_{d}$ as input. 
The term $\log (P /(D+\epsilon)+\epsilon)$ represents a convenient scaling of the ratio between the production $P$ and destruction $D$ terms of the SA model. In the work of Singh et al. (44) the ratio $P / D$ is directly used while in this work a logarithmic scaling is used: this is due to the fact that the values assumed by this ratio are distributed in a wide range which covers several orders of magnitude and some numerical experiments confirmed that the fitting significantly improves with this scaling. Furthermore, both the numerator and the denominator of this quantity can go to zero in the presence of uniform fields or where the turbulence viscosity is zero and so the constant $\epsilon=10^{-5}$ is introduced. Some numerical tests showed that the use of logarithmic scaling improves significantly the fitting of the database with the ANN.

Finally, the adimensional gradient of the modified turbulent viscosity $\log (|\nabla \tilde{\nu}| d /(\nu+$ $\tilde{\nu})+\epsilon)$ is considered. This quantity was not used in (44) and does not appear in the production and destruction terms. However, it appears in the cross production term (the last term of Eq. 4) and allows to identify regions with strong variations in the eddy viscosity. It is normalised with respect to the wall distance and the sum of kinematic and eddy viscosity: this means that this quantity remains well conditioned even when the eddy viscosity tends to zero since the kinematic viscosity prevents the denominator to become zero. Even for this variable the logarithmic scaling was found to be useful to improve the fitting.

\subsection{Choice of the ANN architecture}

After choosing the input features, it is necessary to define the architecture of the ANN. In this work, feedforward ANNs are considered. As far as 


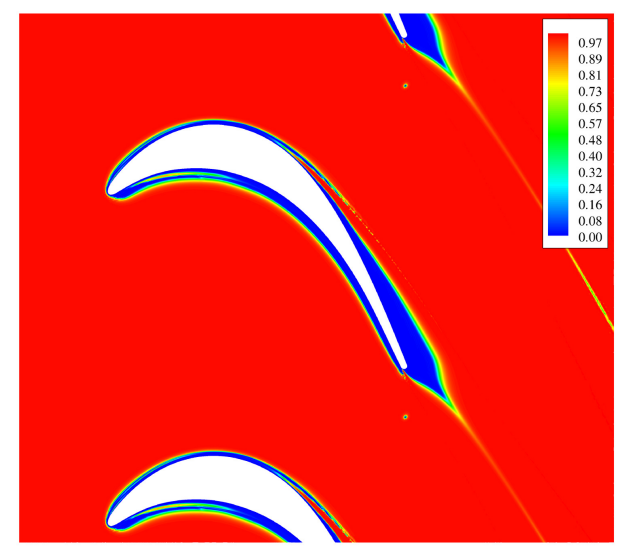

(a)

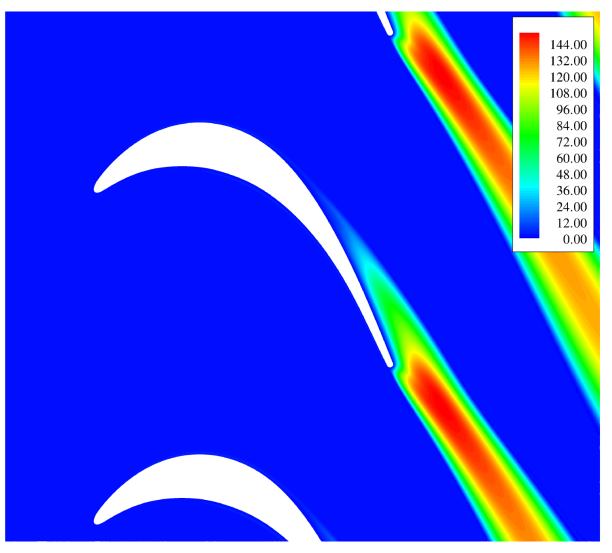

(c)

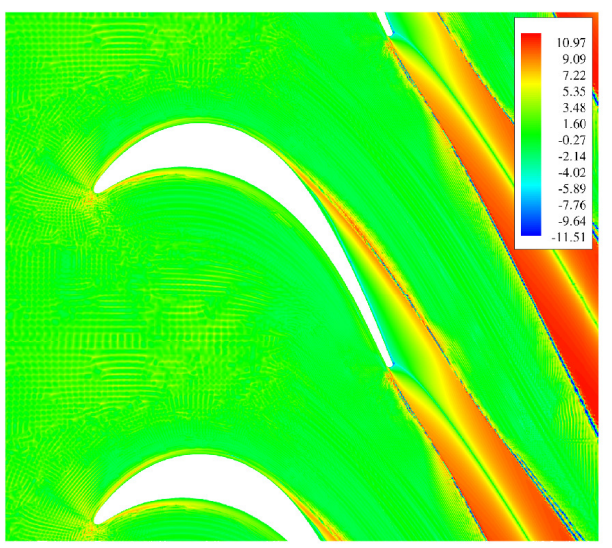

(e)

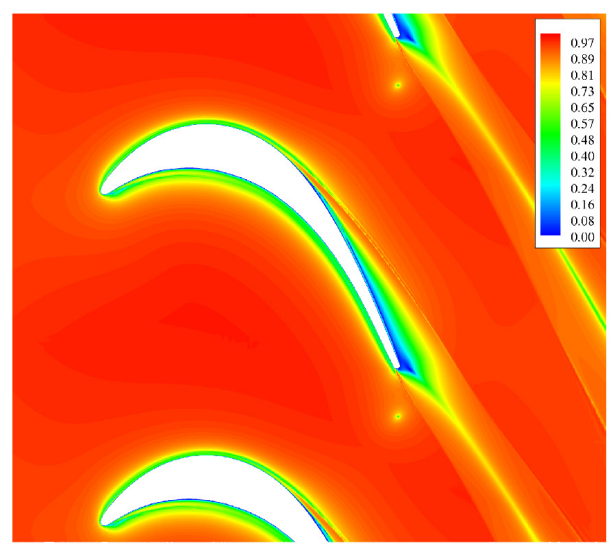

(b)

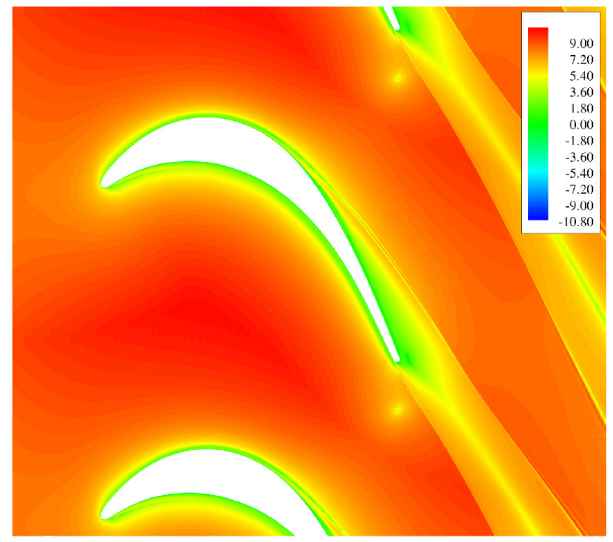

(d)

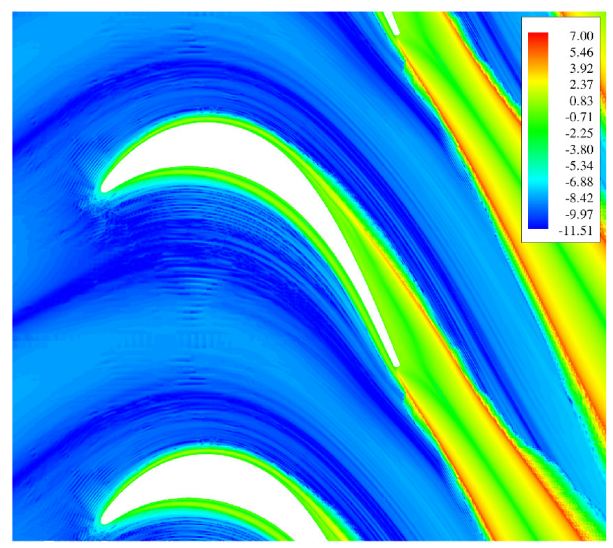

(f)

Figure 6: Input features for the neural network: $f_{d}(\mathrm{a}), f_{d}^{\prime}(\mathrm{b}), \chi(\mathrm{c}), \log \left(\tau / \tau_{r e f}+\epsilon\right)(\mathrm{d})$, $\log (P /(D+\epsilon)+\epsilon)(\mathrm{e}), \log (|\nabla \tilde{\nu}| d /(\nu+\tilde{\nu})+\epsilon)(\mathrm{f})$ 
the activation functions are concerned, a common choice consists in using sigmoid functions for the hidden layers and linear functions for the output layer. However, since the chosen correction factor $h$ is limited in the range $[0,1]$ a sigmoid activation function is adopted also for the output layer: in this way the output of the ANN will be automatically limited in the range $[0,1]$.

Particular care should be taken in choosing the number of hidden layers $n_{H L}$ and the number of neurons per layer $n_{N}=10$. In particular it is necessary to find a compromise between the complexity of the network (which allows to capture the correlations hidden in the database) and its ability to perform predictions outside of the database. When the complexity of the network is increased its ability to reproduce the training database is enhanced because it has more degrees of freedom which can be adjusted to fit the data. However, if too many degrees of freedom are introduced then the network will behave poorly during predictions: this is due to the fact that when too many degrees of freedom are used then the output of the network will show strong oscillations for the points in the parameter space which do not exactly match a training point.

In order to find a suitable network by using a general criteria the following approach is used. First of all, different architectures are considered $(1 \leq$ $\left.n_{H L} \leq 25 \leq n_{N} \leq 40\right)$ and the ability of the networks to fit the database is investigated. Each network is trained in Matlab by means of the LevenbergMarquadt algorithm with a goal function based on the mean squared error. The training is performed by dividing randomly the database in 3 subsets: 
one for training ( $70 \%$ of the data), one for validation ( $15 \%$ of the data) and one for test (15\% of the data). The training set is actually used for the computation of the mean square error and for driving the training process. The validation set is used during the training to verify that the ANN is still able to give good predictions for points which do not belong to the training set: when the validation error tends to increase the training is arrested, even if the training error is still decreasing, in order to limit the problem of overfitting. Finally, the test set is used to monitor the behaviour of the ANN on an external set of data which do not influence the training process (neither in the mean squared error computation nor in the validation checks for the overfitting). An example of training history is reported in Figure 7a in which it can be clearly seen that when the training is stopped the training error was still decreasing but the validation error just started to grow. In Figure 8 it is possible to see the regression plots for the different data sets: in each plot the abscissa represents the reference value in the database while the ordinate represents the approximated value computed by the network. Another approach for avoiding overfitting was also investigated: Bayesan regularisation (53). In Bayesan regularisation the mean square error goal function is augmented by a term which penalises large values of the weights. However, some experiments on the problems considered in this work showed that the splitting of the database in training, validation and test sets allows to achieve a better compromise between fitting and robustness with respect to the Bayesan regularisation.

A sequence of regression plots (on the full database) for the ANN $2 \times 5$, $2 \times 10,2 \times 20$ and $2 \times 40$ are reported in Figure 9: as the complexity of 


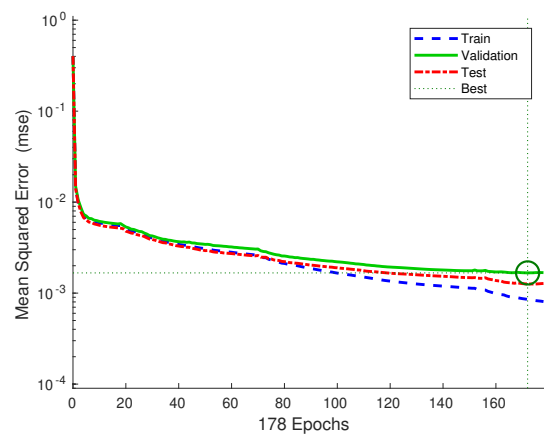

(a)

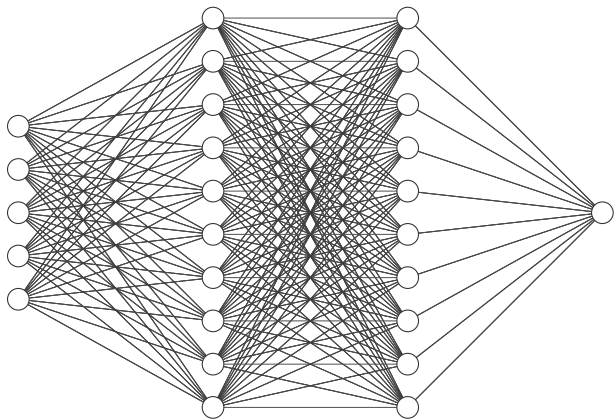

(b)

Figure 7: Training history (a) and architecture (b) for 2x20 ANN

the network is increased its ability to reproduce the database is enhanced as can be cleary seen by the fact that the points tend to assume a distribution centered along the bisector of the quadrant.

In Table 2 the regression coefficient $R$ for different ANN architectures are reported.

\begin{tabular}{|l|c|c|c|c|}
\hline & $n_{N}=5$ & $n_{N}=10$ & $n_{N}=20$ & $n_{N}=40$ \\
\hline$n_{H L}=1$ & 0.799 & 0.831 & 0.865 & 0.897 \\
\hline$n_{H L}=2$ & 0.822 & 0.890 & 0.918 & 0.953 \\
\hline
\end{tabular}

Table 2: Regression coefficient $\mathrm{R}$ for several architectures of the ANN

According to the previous analysis it would seem that the larger is the network the better is the result. This is true for the fitting of the points in the database. However, it is fundamental to investigate the behaviour of the network for points which do not coincide exactly with the points in the database. In order to do this it is possible to run some CFD simulations at 

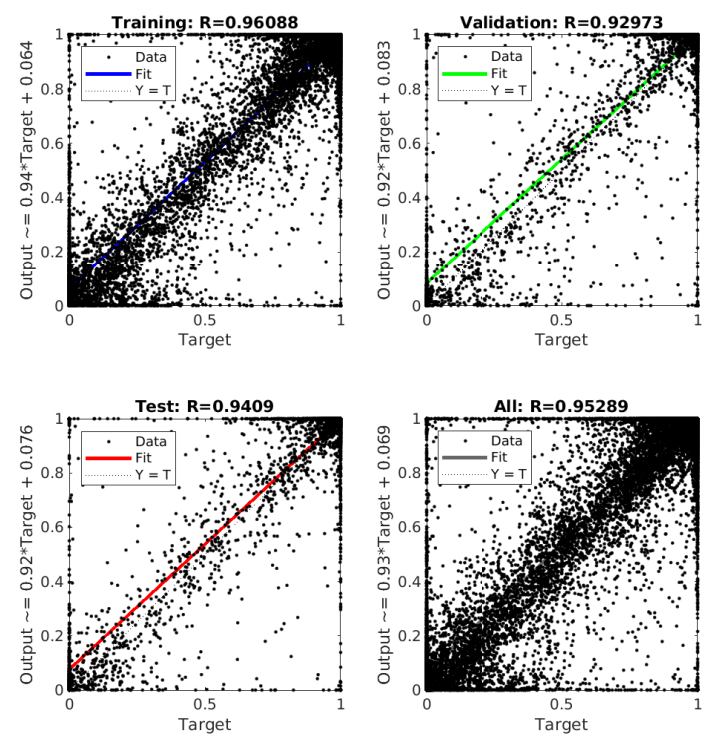

Figure 8: Training, validation and test error for $2 \times 20$ ANN

$R e_{2 s}=8 \cdot 10^{4}$ and $R e_{2 s}=2.5 \cdot 10^{5}$ with the correction term $h$ estimated by the different ANNs. Apparently, this seems a useless check since the database used for the training is built from the optimal solution at these Reynolds number and so one could aspect that the ANN should reproduce perfectly these working conditions. However, it is important to keep in mind that the regression coefficient $R$ is always less than 1: this means that, even if the CFD simulation is initialised with the optimal solution obtained by the adjoint approach, the correction field reproduced by the ANN will not coincide exactly which the optimal one. As a consequence, the CFD solution will evolve towards a new steady solution. This introduces a perturbation in the input features given to the ANN: if the ANN is robust the new steady solution will be close to the optimal one. However, if the ANN is poorly 


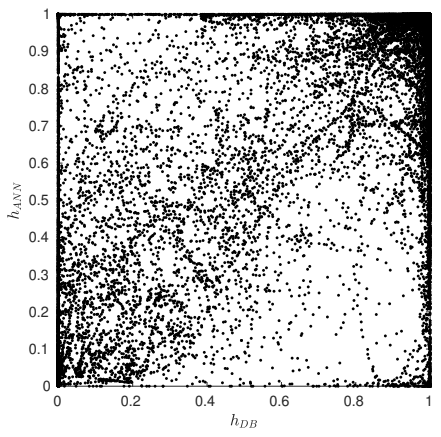

(a)

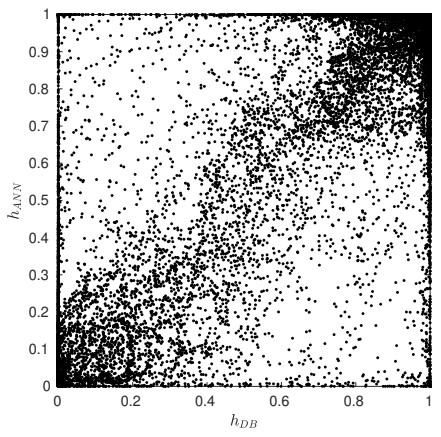

(c)

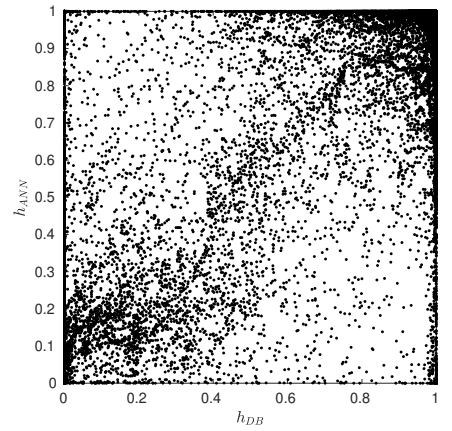

(b)

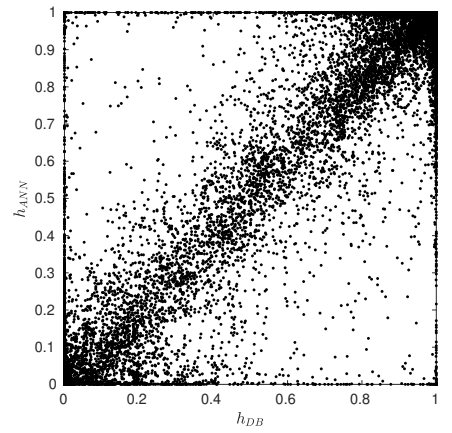

(d)

Figure 9: Regression plots for different ANN architectures: 2x5 (a), 2x10 (b), 2x20 (c), $2 \times 40(d)$ 


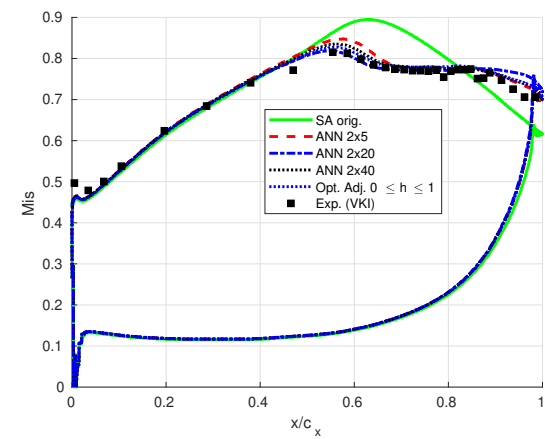

(a)

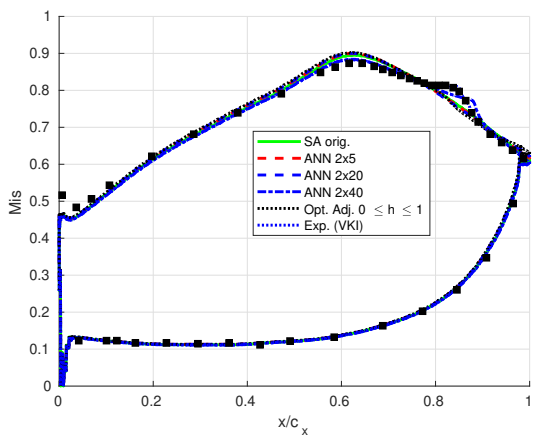

(b)

Figure 10: Comparison of different ANN architectures in terms of wall isentropic Mach number distribution on the T106c at $R e_{2 s}=8 \cdot 10^{4}(\mathrm{a})$ and $R e_{2 s}=2.5 \cdot 10^{5}(\mathrm{~b})$ :

conditioned because an excessive number of neurons has been chosen then the network will give a significantly different response.

This behaviour was verified by checking the wall isentropic Mach number distribution reported in Figure 10 for the ANNs with $2 \times 5,2 \times 10,2 \times 20$ and $2 \times 40$ neurons. It can be seen that the $2 \times 5$ network performs poorly because of its inability to reproduce the database. The networks with $2 \times 10$ and $2 \times 20$ neurons performs significantly better and gives solutions which are very close to the optimal ones. The largest network with $2 \times 40$ neurons starts to show some problems at $R e_{2 s}=2.5 \cdot 10^{5}$ in which it is not able to reproduce the small separation bubble.

According to this analysis, all the predictive simulations reported in the following will be performed by using the $2 \times 20 \mathrm{ANN}$. 


\section{Predictions}

In the previous Section the procedure for choosing the architecture of the ANN is reported. Now, the chosen network is use to perform predictive simulation for working conditions and geometries which were not included in the database. As a first step all the simulations are performed by setting $h(x)=1$, i.e. with the original SA model. Then the obtained steady solution is used to initialise a simulation in which the correction term is computed with the ANN. This approach speed ups the convergence since the ANN is not employed during the strong initial transient at the beginning of the simulation.

Furthermore, the numerical experiments showed that the robustness of the method during predictive simulations can be improved by limiting the input variables to the range used for the training. This is important because the ANN has been trained only on a few steady solutions and so during the transients which can appear in predictive simulations the input features could assume values which were not observed in the training database. In particular, if $h(\boldsymbol{Y})$ represents the ANN approximation of the correction factor and $\boldsymbol{Y}$ is the vector of the five input variables, the modified expression $h(L(\boldsymbol{Y}))$ is used during predictive simulations, where the limiting function $L$ is defined as:

$$
L\left(Y_{i}\right)= \begin{cases}Y_{i} & \text { if } Y_{i}^{\text {min }} \leq Y_{i} \leq Y_{i}^{\max } \\ Y_{i}^{\text {max }} & \text { if } Y_{i}>Y_{i}^{\text {max }} \\ Y_{i}^{\text {min }} & \text { if } Y_{i}<Y_{i}^{\text {min }}\end{cases}
$$

\footnotetext{
Here $Y_{i}^{\min }$ and $Y_{i}^{\max }$ represent the minimum and maximum values of the i-th
} 
input feature observed in the training database.

\subsection{T106c cascade at different Reynolds number}

As a first test, the ANN aumented SA model is used to predict the flow field on the T106c at $R e_{2 s}=1.2 \cdot 10^{5}, 1.6 \cdot 10^{5}$ and $2.1 \cdot 10^{5}$. In this range of Reynolds number a strong variation is observed in the solution due to the transition from open to closed separation. The results related to the wall isentropic Mach number distribution are reported in Figure 11 in which they are compared with the available experimental results and the original SA model. The ANN augmented SA model performs significantly better than the original model and the predictions are quite close to the experiment. Only the solution obtained at $R e_{2 s}=1.2 \cdot 10^{5}$ seems to overpredict the separation.

The results reported in Figure 11 refer to the $M_{i s}$ distribution used in the goal function which drove the field inversion and so it is natural to expect an improvement with respect to the original model. However, the prediction ability of the model was also investigated in terms of mass averaged kinetic losses $\zeta$ and exit angle $\beta_{2}$ in the wake, quantities which were not included in the goal function used for the optimisation.

The average is performed in a control section located $0.465 c_{x}$ behind the trailing edge, where $c_{x}$ is the axial chord, in the same location used for the experimental measurements. The results of these tests are reported in Figure 12. As far as the losses are concerned, both the original SA model and the ANN augmented SA model perform well for high Reynolds values. However, for low Reynolds numbers the original SA model misses completely the separation and so it underpredicts significantly the losses. The ANN augmented SA model shows the correct trend and is quite close to the experimental re- 


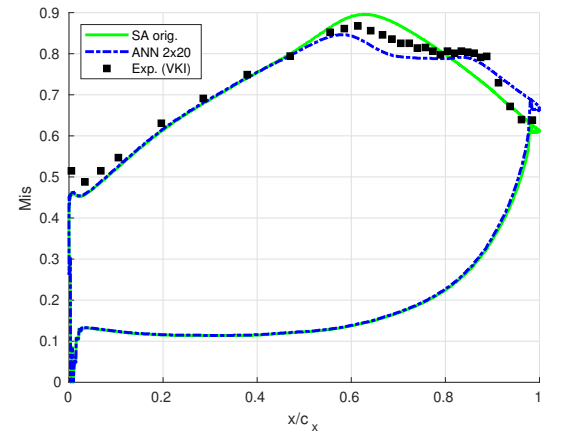

(a)

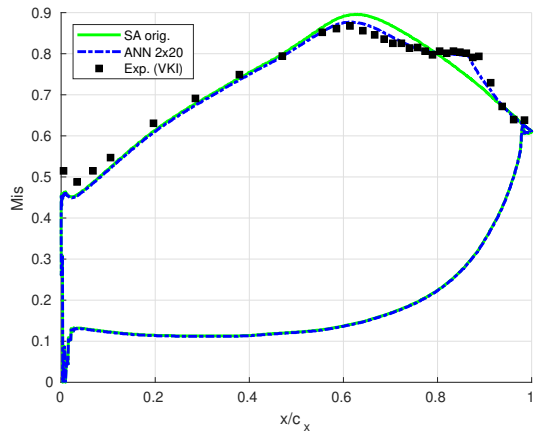

(b)

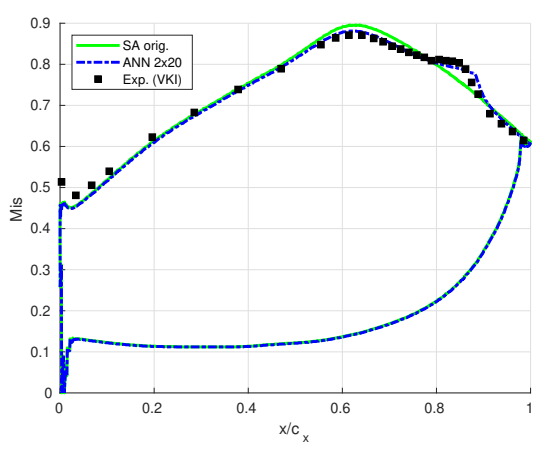

(c)

Figure 11: Wall isentropic Mach number distribution: predictions at $\operatorname{Re}_{2 s}=1.2 \cdot 10^{5}$ (a), $R e_{2 s}=1.6 \cdot 10^{5}(\mathrm{~b})$ and $R e_{2 s}=2.1 \cdot 10^{5}$ (c) 
sults at $R e_{2 s}=8 \cdot 10^{4}$ (for which the optimisation was performed). The plot shows also the results obtained by Benyahia et al. (54) with the SST- $\gamma-R e_{\theta}$ model based on the correlations proposed by (55), by Pacciani et al.(56) with the $k-\omega$ model coupled with a transport equation for the laminar kinetic energy and by Babajee (50) with the SST- $\gamma-R e_{\theta}$ model $(57 ; 58)$. The boundary condition for the turbulent kinetic energy equation which appears in the SST model is clearly defined by the experimental inlet turbulence intensity (0.9\%). However, the SST model requires also an inlet boundary condition for the $\omega$ equation which is usually prescribed by defining an inlet turbulence Reynolds number $\left(R e_{T}\right)$. Babajee performed a study on the choice of the inlet value for $R e_{T}$ : in particular he found the optimal value of $R e_{T}$ which fits the experimental turbulence decay in the wind tunnel without the cascade. However, when this value is imposed at the inlet, the SST- $\gamma-R e_{\theta}$ model is not able to predict accurately the separation. For this reason he performed a parametric study changing $R e_{T}$ in order to match at best the experimental results on the T106c. For this reason, the plot shows two set of results related to the SST- $\gamma-R e_{\theta}$ model: the results with the boundary condition which is coherent with the physical decay of turbulence in the wind tunnel $\left(\operatorname{Re}_{T}=T D\right)$ and the results with an alternative value which gives better predictions $\left(R e_{T}=0.01\right)$.

As far as the average exit angle is concerned, the ANN augmented SA model shows a better behaviour than the original SA model at low Reynolds numbers while the two models give similar results at higher Reynolds numbers. It is interesting to note that the asymptotic value of the exit angle for high values of Reynolds number presents an offset between experimental and nu- 


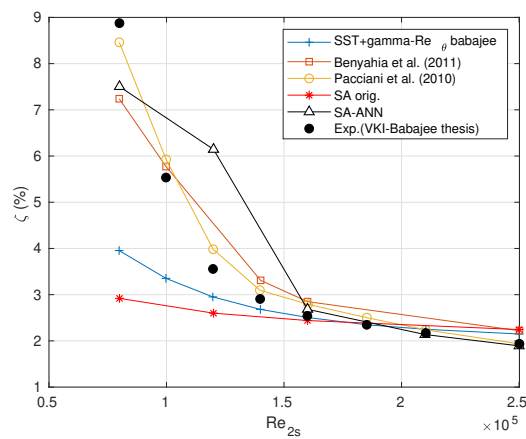

(a)

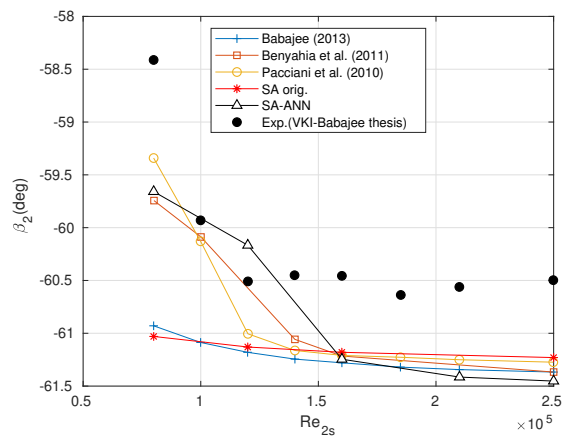

(b)

Figure 12: Average losses and exit angle for T106c cascade: comparison between original SA model, SA-ANN model and experimental results

merical results. However, this offset was observed also by other results in the literature as shown by the SST- $\gamma-R_{\theta}$ results from (50).

\subsection{T2 cascade}

The prediction ability of the ANN augmented SA model is investigated also on another geometry, the T2 cascade. The simulations are carried out with a third order accurate DG scheme on a mesh with 59453 elements, corresponding to 356718 degrees of freedom per equation. The mesh resolution at wall and in the wake region is the same used for the T106c, since both cascades are investigated at similar values of Reynolds number. The T2 airfoil was designed at the VKI for the same velocity triangles of the T106 (inlet angle $\alpha=32.7^{\circ}$ ) but it is characterised by a larger pitch-to-chord ratio (1.05) and an increased diffusion rate along the rear suction side (50). Also the Zweifel number is larger $(\Psi=1.46)$ with respect to the T106 $(\Psi=1.24)$. The isentropic exit Mach number is set to $M_{2 s}=0.65$. 
In Figure 13 and 14 the Mach number field at $R e_{2 s}=1.2 \cdot 10^{5}$ and $2.1 \cdot 10^{5}$ is reported for the original SA model and for the ANN augmented SA model. The plots show clearly the presence of a open separation at $R e_{2 s}=1.2 \cdot 10^{5}$ and a closed separation at $R e_{2 s}=2.1 \cdot 10^{5}$.

Finally, in Figure 15 the predicted wall isentropic Mach number distribution is reported as a function of the curvilinear coordinate $s$ along the blade surface, normalised with respect to the curvilinear length of the blade $\left(s_{0}\right)$. The ANN augmented SA model shows significant improvements with respect to the baseline SA model and gives good results also with respect to the SST$\gamma-\operatorname{Re}_{\theta}$ results from (50).

Finally, the models are evaluated in terms of mass averaged exit kinetic losses and angle, as reported in Figure 16. As observed for the T106c, even in this case the ANN augmented SA model outperforms the original SA model at low Reynolds numbers. It is interesting to note that the numerical results obtained in the present work presents an offset in $\beta_{2}$ with respect to the experimental results, offset which is not observed in the results obtained from the SST- $\gamma-\operatorname{Re}_{\theta}$ model. This could be a limitation of the SA model which is inherited by the augmented model: future work will be devoted to apply the field inversion approach to other RANS models to verify whether this limitation persists.

\section{Conclusions}

The potential of the field inversion approach was investigated for the augmentation of a RANS model used in the simulation of turbomachinery flows. In particular the approach was applied to the original SA model and the 


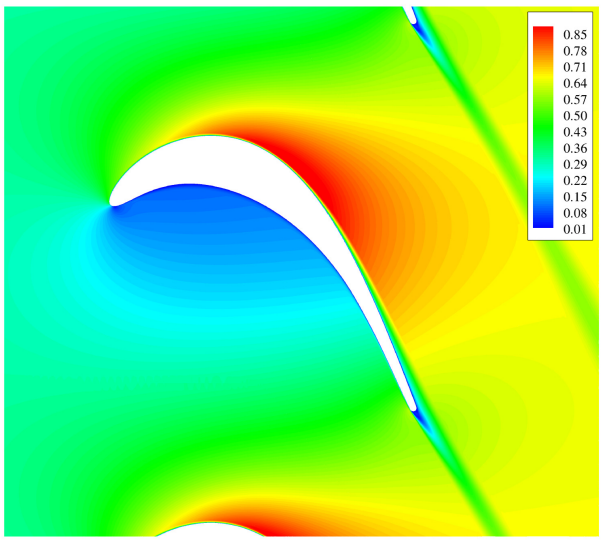

(a)

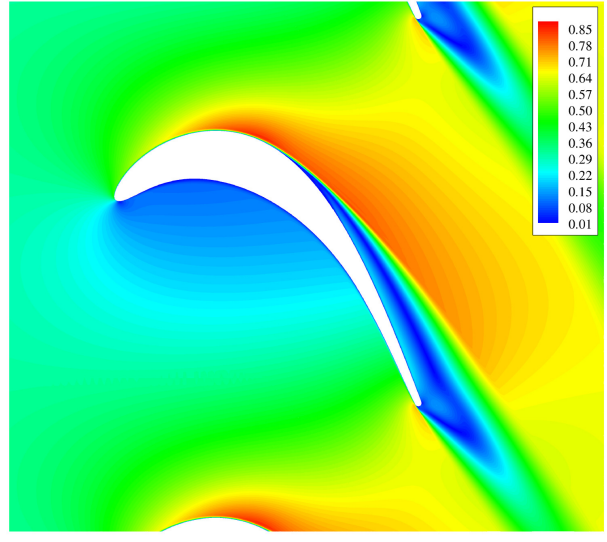

(b)

Figure 13: Mach field for the T2 cascade at $R e_{2 s}=1.2 \cdot 10^{5}$ with the original SA model (a) and with the ANN-SA model (b)

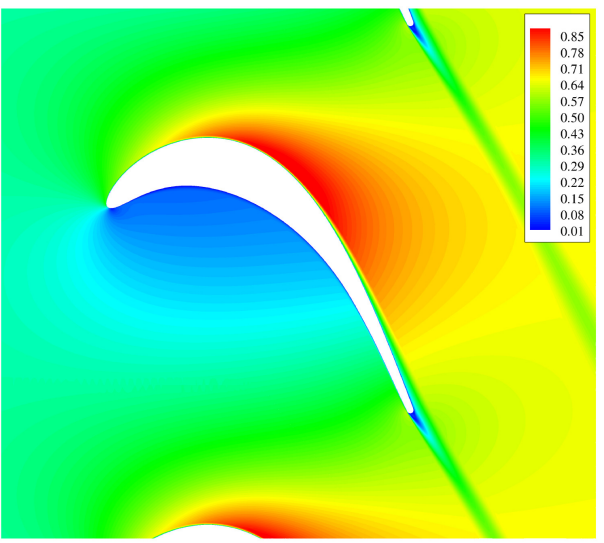

(a)

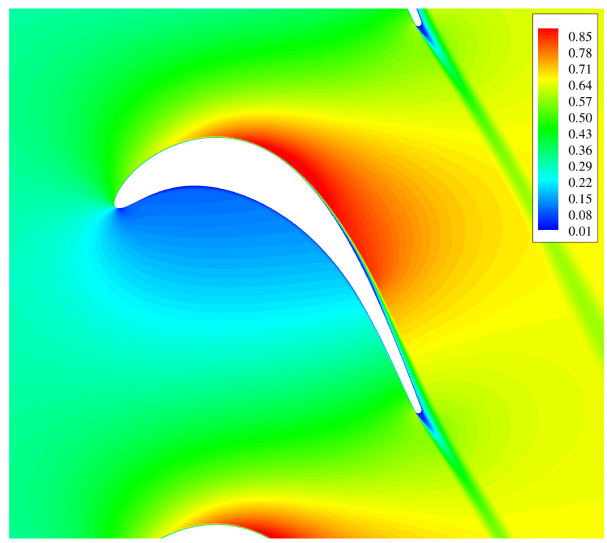

(b)

Figure 14: Mach field for the T2 cascade at $R e_{2 s}=2.1 \cdot 10^{5}$ with the original SA model (a) and with the ANN-SA model (b) 


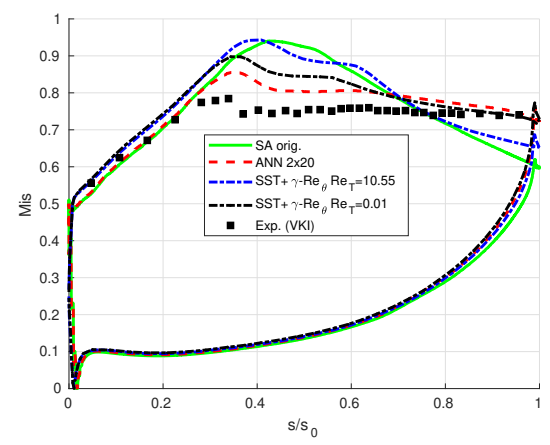

(a)

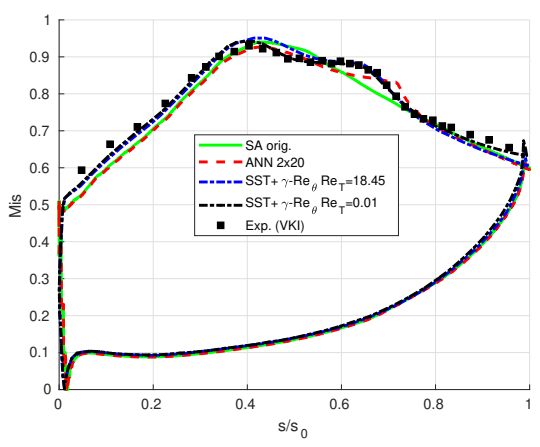

(b)

Figure 15: Mis distribution for T2 cascade at $\operatorname{Re}_{2 s}=1.2 \cdot 10^{5}(\mathrm{a})$ and $\operatorname{Re}_{2 s}=2.1 \cdot 10^{5}(\mathrm{~b})$ : comparison between original SA model, SA-ANN model and experimental results

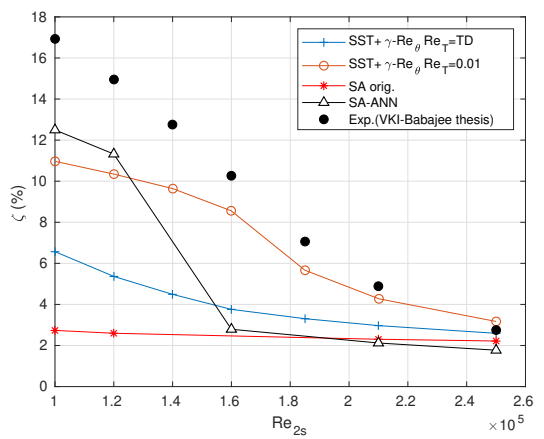

(a)

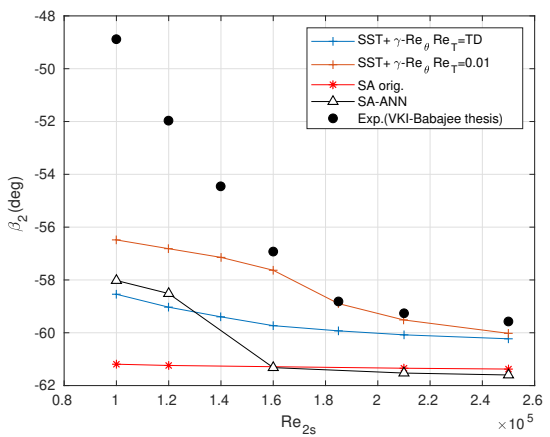

(b)

Figure 16: Average losses and exit angle for T2 cascade: comparison between original SA model, SA-ANN model and experimental results 
attention is focused on transitional flows with separation in low pressure gas turbines. Since the original model is not suited for this kind of flows, the field inversion approach is used to develop a local correction of the production term which acts like an intermittency correction for transitional flows. The correction factor is then expressed by means of an ANN as a function of some physical quantities in order to generalise the model. An investigation has been carried out on the definition of the input features which are improved with respect to the original definitions suggested in the literature. A convergence study is carried out to choose the architecture of the ANN in order to underline the problem of overfitting. The ability of the ANN augmented SA model to compute low Reynolds number flow fields in low pressure gas turbine cascades is investigated by performing actual predictions at different Reynolds numbers and on a different geometry with respect to the one used for the field inversion. Furthermore, a new expression of the correction term is proposed in order to limit its value in a finite range: this, together with the introduction of a limiting on input features, significantly improves the robustness of the approach during transients and in predictions.

The results seem promising and are substantially better than the results provided by the original model. They also appears satisfactory if compared to the results obtained by a significantly more complex four equation model $\left(\mathrm{SST}-\gamma-R e_{\theta}\right)$. In particular, even if the goal function used for the field inversion is based only on the wall isentropic Mach number, the ANN augmented model shows improvements also in terms of average losses and exit angle in the wake.

Future work will be devoted to the application of the field inversion approach 
to other RANS models. Furthermore, possible alternatives to the use of an ANN will be investigated for achieving a better fitting of the database with a good level of robustness in predictions.

\section{Acknowledgements}

Computational resources were provided by HPC@POLITO, a project of Academic Computing within the Department of Control and Computer Engineering at the Politecnico di Torino (http://www.hpc.polito.it).

We aknowledge the CINECA award under the ISCRA initiative, for the availability of high performance computing resources and support. The simulations were performed on the Marconi Tier-0 System for the Project FIRST.

\section{References}

[1] S. Russo, P. Luchini, A fast algorithm for the estimation of statistical error in dns (or experimental) time averages, Journal of Computational Physics 347 (2017) 328-340.

[2] R. D. Sandberg, V. Michelassi, The current state of high-fidelity simulations for main gas path turbomachinery components and their industrial impact, Flow, Turbulence and Combustion 102 (4) (2019) 797-848.

[3] X. Xie, M. Mohebujjaman, L. G. Rebholz, T. Iliescu, Data-driven filtered reduced order modeling of fluid flows, SIAM Journal on Scientific Computing 40 (3) (2018) B834-B857. 
[4] R. Dupuis, J.-C. Jouhaud, P. Sagaut, Surrogate modeling of aerodynamic simulations for multiple operating conditions using machine learning, AIAA Journal 56 (9) (2018) 3622-3635.

[5] L. Margheri, M. Meldi, M. V. Salvetti, P. Sagaut, Epistemic uncertainties in rans model free coefficients, Computers \& Fluids 102 (2014) $315-335$.

[6] A. Ferrero, A. Iollo, F. Larocca, Reduced order modelling for turbomachinery shape design, International Journal of Computational Fluid Dynamics (2019) 1-12.

[7] A. Ferrero, A. Iollo, F. Larocca, Global and local pod models for the prediction of compressible flows with dg methods, International Journal for Numerical Methods in Engineering 116 (5) (2018) 332-357.

[8] M. Bergmann, A. Ferrero, A. Iollo, E. Lombardi, A. Scardigli, H. Telib, A zonal galerkin-free pod model for incompressible flows, Journal of Computational Physics 352 (2018) 301-325.

[9] M. Raissi, G. E. Karniadakis, Hidden physics models: Machine learning of nonlinear partial differential equations, Journal of Computational Physics 357 (2018) 125-141.

[10] J.-X. Wang, J.-L. Wu, H. Xiao, Physics-informed machine learning approach for reconstructing reynolds stress modeling discrepancies based on dns data, Physical Review Fluids 2 (3) (2017) 034603.

[11] J. Weatheritt, R. Sandberg, A novel evolutionary algorithm applied to 
algebraic modifications of the rans stress-strain relationship, Journal of Computational Physics 325 (2016) 22-37.

[12] J. Weatheritt, R. Pichler, R. D. Sandberg, G. Laskowski, V. Michelassi, Machine learning for turbulence model development using a high-fidelity hpt cascade simulation, in: ASME Turbo Expo 2017: Turbomachinery Technical Conference and Exposition, American Society of Mechanical Engineers Digital Collection, 2017.

[13] K. Duraisamy, Z. J. Zhang, A. P. Singh, New approaches in turbulence and transition modeling using data-driven techniques, in: 53rd AIAA Aerospace Sciences Meeting, 2015, p. 1284.

[14] E. J. Parish, K. Duraisamy, A paradigm for data-driven predictive modeling using field inversion and machine learning, Journal of Computational Physics 305 (2016) 758-774.

[15] F. Gibou, D. Hyde, R. Fedkiw, Sharp interface approaches and deep learning techniques for multiphase flows, Journal of Computational Physics 380 (2019) 442-463.

[16] H. Patel, A. Panda, J. Kuipers, E. Peters, Computing interface curvature from volume fractions: A machine learning approach, Computers \& Fluids 193 (2019) 104263.

[17] A. Takbiri-Borujeni, M. Ayoobi, Application of physics-based machine learning in combustion modeling (2019).

[18] S. Bhalla, M. Yao, J.-P. Hickey, M. Crowley, Compact representation of 
a multi-dimensional combustion manifold using deep neural networks, in: European Conference on Machine Learning, 2019.

[19] X. Zhu, Z. Cai, J. Wu, Y. Cheng, Q. Huang, Convolutional neural network based combustion mode classification for condition monitoring in the supersonic combustor, Acta Astronautica 159 (2019) 349-357.

[20] Y.-P. Zhao, Q.-K. Hu, J.-G. Xu, B. Li, G. Huang, Y.-T. Pan, A robust extreme learning machine for modeling a small-scale turbojet engine, Applied energy 218 (2018) 22-35.

[21] Z. Liu, I. A. Karimi, Gas turbine performance prediction via machine learning, Energy (2019) 116627.

[22] K. Duraisamy, G. Iaccarino, H. Xiao, Turbulence modeling in the age of data, Annual Review of Fluid Mechanics 51 (2019) 357-377.

[23] S. R. Allmaras, F. T. Johnson, Modifications and clarifications for the implementation of the spalart-allmaras turbulence model, in: Seventh international conference on computational fluid dynamics (ICCFD7), 2012, pp. 1-11.

[24] A. Ferrero, A. Iollo, F. Larocca, Rans closure by artificial neural networks, in: 13 th European Conference on Turbomachinery Fluid dynamics \& Thermodynamics, EUROPEAN TURBOMACHINERY SOCIETY, 2019.

[25] R. Hartmann, P. Houston, Adaptive discontinuous galerkin finite element methods for the compressible euler equations, Journal of Computational Physics 183 (2) (2002) 508-532. 
[26] J.-F. Remacle, J. E. Flaherty, M. S. Shephard, An adaptive discontinuous galerkin technique with an orthogonal basis applied to compressible flow problems, SIAM review 45 (1) (2003) 53-72.

[27] G. Zenoni, T. Leicht, A. Colombo, L. Botti, An agglomeration-based adaptive discontinuous galerkin method for compressible flows, International Journal for Numerical Methods in Fluids 85 (8) (2017) 465-483.

[28] A. Ferrero, F. Larocca, V. Bernaschek, Unstructured discretisation of a non-local transition model for turbomachinery flows, Advances in aircraft and spacecraft science 4 (5) (2017) 555-571.

[29] A. Burbeau, P. Sagaut, A dynamic p-adaptive discontinuous galerkin method for viscous flow with shocks, Computers \& fluids 34 (4-5) (2005) $401-417$.

[30] G. Giorgiani, S. Fernández-Méndez, A. Huerta, Hybridizable discontinuous galerkin p-adaptivity for wave propagation problems, International Journal for Numerical Methods in Fluids 72 (12) (2013) 1244-1262.

[31] E. Ampellio, F. Bertini, A. Ferrero, F. Larocca, L. Vassio, Turbomachinery design by a swarm-based optimization method coupled with a cfd solver, Advances in aircraft and spacecraft science 3 (2) (2016) 149.

[32] C. Eskilsson, An hp-adaptive discontinuous galerkin method for shallow water flows, International Journal for Numerical Methods in Fluids 67 (11) (2011) 1605-1623.

[33] A. Ferrero, F. Larocca, Adaptive cfd schemes for aerospace propulsion, 
in: Journal of Physics: Conference Series, Vol. 841, IOP Publishing, 2017, p. 012017.

[34] N. Chalmers, G. Agbaglah, M. Chrust, C. Mavriplis, A parallel hpadaptive high order discontinuous galerkin method for the incompressible navier-stokes equations, Journal of Computational Physics: X 2 (2019) 100023.

[35] C. Geuzaine, J.-F. Remacle, Gmsh: A 3-d finite element mesh generator with built-in pre-and post-processing facilities, International journal for numerical methods in engineering 79 (11) (2009) 1309-1331.

[36] M. Lange, M. G. Knepley, G. J. Gorman, Flexible, scalable mesh and data management using petsc dmplex, in: Proceedings of the 3rd International Conference on Exascale Applications and Software, University of Edinburgh, 2015, pp. 71-76.

[37] S. Balay, S. Abhyankar, M. Adams, J. Brown, P. Brune, K. Buschelman, L. Dalcin, A. Dener, V. Eijkhout, W. Gropp, et al., Petsc users manual (2019).

[38] F. Bassi, L. Botti, A. Colombo, D. A. Di Pietro, P. Tesini, On the flexibility of agglomeration based physical space discontinuous galerkin discretizations, Journal of Computational Physics 231 (1) (2012) 45-65.

[39] S. Osher, F. Solomon, Upwind difference schemes for hyperbolic systems of conservation laws, Mathematics of computation 38 (158) (1982) 339374. 
[40] M. Pandolfi, A contribution to the numerical prediction of unsteady flows, AIAA journal 22 (5) (1984) 602-610.

[41] A. Ferrero, F. Larocca, G. Puppo, A robust and adaptive recovery-based discontinuous galerkin method for the numerical solution of convectiondiffusion equations, International Journal for Numerical Methods in Fluids 77 (2) (2015) 63-91.

[42] F. Bassi, L. Botti, A. Colombo, A. Crivellini, N. Franchina, A. Ghidoni, S. Rebay, Very high-order accurate discontinuous galerkin computation of transonic turbulent flows on aeronautical configurations, in: ADIGMA-A European Initiative on the Development of Adaptive Higher-Order Variational Methods for Aerospace Applications, Springer, 2010, pp. 25-38.

[43] A. Ferrero, F. Larocca, Feedback filtering in discontinuous galerkin methods for euler equations, Progress in Computational Fluid Dynamics, an International Journal 16 (1) (2016) 14-25.

[44] A. P. Singh, S. Medida, K. Duraisamy, Machine-learning-augmented predictive modeling of turbulent separated flows over airfoils, AIAA Journal (2017) 2215-2227.

[45] C. M. Bishop, Training with noise is equivalent to tikhonov regularization, Neural computation 7 (1) (1995) 108-116.

[46] L. Hascoet, V. Pascual, The tapenade automatic differentiation tool: Principles, model, and specification, ACM Transactions on Mathematical Software (TOMS) 39 (3) (2013) 20. 
[47] K. Duraisamy, P. Durbin, Transition modeling using data driven approaches, in: CTR Summer Program, 2014, p. 427.

[48] J. Michalek, M. Monaldi, T. Arts, Aerodynamic performance of a very high lift low pressure turbine airfoil (t106c) at low reynolds and high mach number with effect of free stream turbulence intensity, Journal of Turbomachinery 134 (6) (2012) 061009.

[49] 2nd international workshop on high-order cfd method, https://www.dlr.de/as/desktopdefault.aspx/tabid-8170/13999read$35550 /$, accessed : $2019-12-02$.

[50] J. Babajee, Detailed numerical characterization of the separationinduced transition, including bursting, in a low-pressure turbine environment, Ph.D. thesis (2013).

[51] J. Hourmouziadis, Aerodynamic design of low pressure turbines, AGARD, Blading Design for Axial Turbomachines 40 p(SEE N 89-27661 22-07) (1989).

[52] P. R. Spalart, S. Deck, M. L. Shur, K. D. Squires, M. K. Strelets, A. Travin, A new version of detached-eddy simulation, resistant to ambiguous grid densities, Theoretical and computational fluid dynamics 20 (3) (2006) 181.

[53] F. D. Foresee, M. T. Hagan, Gauss-newton approximation to bayesian learning, in: Proceedings of International Conference on Neural Networks (ICNN'97), Vol. 3, IEEE, 1997, pp. 1930-1935. 
[54] A. Benyahia, L. Castillon, R. Houdeville, Prediction of separationinduced transition on high lift low pressure turbine blade, in: ASME 2011 Turbo Expo: Turbine Technical Conference and Exposition, American Society of Mechanical Engineers Digital Collection, 2011, pp. 18351846.

[55] C. Content, R. Houdeville, Application of the $\gamma$-r $\theta$ laminar-turbulent transition model in navier-stokes computations, in: 40th Fluid Dynamics Conference and Exhibit, 2010, p. 4445.

[56] R. Pacciani, M. Marconcini, A. Arnone, F. Bertini, A cfd study of low reynolds number flow in high lift cascades, in: ASME Turbo Expo 2010: Power for Land, Sea, and Air, American Society of Mechanical Engineers Digital Collection, 2010, pp. 1525-1534.

[57] F. R. Menter, R. B. Langtry, S. Likki, Y. Suzen, P. Huang, S. Völker, A correlation-based transition model using local variables - part i: model formulation, Journal of turbomachinery 128 (3) (2006) 413-422.

[58] R. B. Langtry, F. Menter, S. Likki, Y. Suzen, P. Huang, S. Völker, A correlation-based transition model using local variables-part ii: test cases and industrial applications, Journal of Turbomachinery 128 (3) (2006) 423-434. 